NASA Technical Memorandum 102836

\title{
On Turbulent Spots In Plane Poiseuille Flow
}

\section{Dan S. Henningson and John Kim}

February 1992

(NASA-TM-102836) ON TURBULENT SPOTS IN

N92-25136

PLANE POISEUILLE FLOW (NASA) $40 \mathrm{P}$ CSCL 200

Unclas

G3/34 0078063

\section{N/SA}


NASA Technical Memorandum 102836

\section{On Turbulent Spots In Plane Poiseuille Flow}

Dan S. Henningson, Massachusetts Institute of Technology, Cambridge, Massachusetts John Kim, Ames Research Center, Moffett Field, California

February 1992

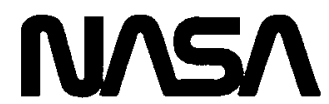

National Aeronautics and

Space Administration 


\title{
ON TURBULENT SPOTS IN PLANE POISEUILLE FLOW
}

\author{
Dan S. Henningson \\ Department of Mathematics, MIT, Cambridge MA 02139 \\ and \\ John Kim \\ NASA Ames Research Center, Moffett Field, CA 94035
}

Turbulence characteristics inside a turbulent spot in plane Poiseuille flow are investigated by analyzing a database obtained from a direct numerical simulation. The spot is found to consist of two distinct regions - a turbulent area and a wave area. The flow inside the turbulent area has a strong resemblance to that found in the fully developed turbulent channel. Suitably defined mean and rms fluctuations as well as the internal shear-layer structures are found to be similar to the turbulent counterpart. In the wave area the inflexional mean spanwise profiles cause a rapid growth of oblique waves, which break down to turbulence. The breakdown process of the oblique waves is reminiscent of the secondary instability observed during transition to turbulence in channel and boundary-layer flows. Other detailed characteristics associated with the Poiseuille spot are presented and are compared with experimental results.

\section{Introduction}

The transition of a laminar flow to a turbulent state has been studied extensively over the years. The interested reader is referred to Maslowe, 1981 and Herbert, 1988 for a review of the subject. The transition process can take several different routes depending on the initial disturbance environment. In this paper we study the transition to turbulence from localized disturbances and concentrate on the later stages when a turbulent spot has developed. We briefly review the breakdown of two-dimensional waves in the introduction, since it is found that certain characteristics of the breakdown of waves found in the Poiseuille flow spot are similar to those found for two-dimensional waves.

\subsection{Two-dimensional waves and secondary instability}

Experimentally observed growth rates of two-dimensional instability waves (Klebanoff, Tidstrom, \& Sargent 1962; Nishioka, Iida, \& Ichikawa 1976) are in good agreement with those predicted by the linearized Navier-Stokes equation. When the amplitude of the primary two-dimensional instability wave (Tollmien-Schlichting, or $\mathrm{T}-\mathrm{S}$ wave) becomes finite, it becomes unstable to three-dimensional disturbances. 
The initially two-dimensional wave develops into a three-dimensional structure in the form of peaks and valleys in the spanwise direction. The peaks are seen to move ahead of the valleys to form shear layers located over $\Lambda$-vortex structures (Kovasznay, Komoda \& Vasudeva, 1962). The appearance of three-dimensionality is referred to as the secondary instability (Herbert, 1988). A number of numerical simulations have been performed to investigate the secondary instability. Orszag \& Patera (1983), for instance, calculated the stability of finite amplitude two-dimensional waves to infinitesimal oblique waves. They found large growth rates for a wide class of wall-bounded shear flows.

\subsection{Localized disturbances}

Natural transition rarely starts with growth of two-dimensional waves. Instead disturbances often appear initially localized both in space and time. If the Reynolds number is close to the critical Reynolds number, a localized disturbance quickly develops into a wave packet. Gaster (1975) calculated the development of a localized pulsed perturbation in a Blasius boundary layer by summing the least stable mode over all wave numbers, assuming that they were all initially equally excited. His result showed a swept-back wave packet that dispersed in both the streamwise and spanwise directions. Experiments by Gaster \& Grant (1975) on the development of a wave packet showed the same space-time development as in the calculation. In addition to the wave packet, there are other effects present in a three-dimensional disturbance. In the inviscid case, Landahl (1980) showed that such disturbances may be algebraically unstable, leading to a disturbance energy growing linearly in time. The increase comes about because the disturbed region will elongate in the streamwise direction with a nondecaying streamwise perturbation. Verification of these effects in viscous flows have recently been made by Henningson, Johansson \& Lundbladh (1990). Their numerical simulations of the development of localized disturbances also showed that the nonlinear growth was dominated by classical wave number summations, primarily involving wave modes with zero streamwise wavenumber, i.e., disturbances elongated in the streamwise direction. Experiments by Chambers \& Thomas (1983) on the early stages of turbulent spots confirm the importance of these effects. They found that longitudinal streaks in front of the wave packet were responsible for the spreading of the disturbance, and that the wave part of the disturbance was not a primary cause of the breakdown.

\subsection{Turbulent spots}

When a localized disturbance evolves for a long time in an unstable flow, or the disturbance is large enough in a linearly stable one, the breakdown of the disturbance results in a localized region of turbulence, i.e., a turbulent spot. Emmons 
(1951) was the first to report a turbulent spot, in a water table study. A majority of subsequent studies, however, have concentrated on boundary-layer and Poiseuille spots.

\section{Boundary-layer spots}

Schubauer \& Klebanoff (1955), in their study of boundary-layer transition, investigated the characteristics of turbulent spots. They found that the leading arrowhead of the boundary-layer spot propagated with about $90 \%$ of the free-stream velocity, and that it consisted of an overhang with laminar fluid underneath. The straight rear interface moved with about half the free-stream velocity and the spot was found to spread with a half-angle of about $10^{\circ}$. Wygnanski, Sokolov \& Freedman (1976) showed that the streamwise mean velocity inside the spot has a logarithmic character, typical of a fully developed turbulent boundary layer, and that the mean spanwise velocity was directed outward in the turbulent region. Measurements by Johansson, Her \& Haritonidis (1987) further demonstrated that both turbulence statistics and turbulence structure inside a turbulent spot are similar to those in a fully developed turbulent boundary layer.

The lateral spreading of a spot is known to be too large to be explained by entrainment arguments. Gad-el-Hak, Blackwelder \& Riley (1981) proposed an instability mechanism they referred to as growth by destabilization to be responsible. They found that new turbulence, which added to the spanwise extent of the spot, broke down because the outside region had been destabilized. The destabilization mechanism, however, was not clear in their study. Wygnanski, Haritonidis \& Kaplan (1979) found T-S waves trailing the spanwise wingtips of the spot, and conjectured that the spreading was caused by the observed breakdown of these waves. A recent study by Glezer, Katz \& Wygnanski (1989) considered this packet in more detail and they found that in certain instances the breakdown of the waves were seen to add to the turbulent part of the spot. Chambers \& Thomas (1983), however, argued that the trailing packet was only a remnant of the waves caused by the initial disturbance, and that it played no significant role in the spreading or breakdown of the boundary-layer spot itself.

\section{Poiseuille flow spots}

Turbulent spots are also found in plane Poiseuille flow. Carlson, Widnall \& Peeters (1982) found that those spots had an arrowhead pointing in the upstream direction, in contrast to the boundary-layer spot. Around the spot they found a wave pattern not seen in the other types of spots. Oblique waves were seen at the spanwise wingtips and trailing the turbulent region. Alavyoon, Henningson \& Alfredsson (1986) also investigated the Poiseuille flow spot. They showed that the propagation velocities varied with Reynolds number, with typical values of $80 \%$ and $50 \%$ of 
the centerline velocity for the front and the trailing interface, respectively. The spreading half-angle of the Poiseuille spot was found to increase with Reynolds number, but had a typical value of about $10^{\circ}$. A direct numerical simulation of the Poiseuille spot using the full Navier-Stokes equations by Henningson, Spalart \& Kim (1987) agrees well with the findings of the experiments. Thus, the propagation velocities and spreading angle of the spots in the boundary layer and Poiseuille flow are similar, whereas the shape and wave structure exhibit differences.

The waves at the wingtips of the Poiseuille spot were experimentally investigated by Henningson \& Alfredsson (1987). They consisted of the least stable Orr-Sommerfeld mode, and propagated into the turbulent region as they broke down. Stability calculations of the measured laminar velocity field outside the wingtips showed that those regions were destabilized. Further investigation of the waves by Henningson (1989), using velocity fields obtained from the numerical simulation of Henningson, Spalart, \& Kim (1987), has shown that they grow because of a combination of "cross flow" instability and wave energy focusing. The cross flow instability results from the presence of inflexional spanwise mean velocity profiles in the wingtip region, and the wave energy focusing is associated with converging group velocity rays. An attempt to model the wave field surrounding the spot has recently been done by $\mathrm{Li} \&$ Widnall (1989). They calculated the wave field outside a moving patch of Reynolds stress and obtained waves in qualitative agreement with the experiments. The waves in their model, however, were stationary in a frame of reference moving with the spot, and the speed of the patch was taken substantially lower than the observed propagation velocity of the spot.

The objective of this paper is to investigate the characteristics of the turbulence inside a fully developed Poiseuille spot. We shall address to what extent the flow characteristics inside the turbulent spot resemble those in turbulent channel flow. Turbulence statistics as well as turbulence structure in the spot will be examined. This paper will complement the work of Henningson (1989), who reported the mechanism for growth of the wingtip waves. Here we will report on the breakdown of those waves.

A brief description of the numerical method and initial condition used in the simulation is given in $\S 2$. In $\S 3$ the instantaneous and mean velocity fields obtained from the simulation are investigated, including the velocity fields associated with the wingtip waves. Turbulence statistics in the spot, such as the r.m.s. values and Reynolds stress, are presented in $\S 4$, along with an analysis of typical flow structures found in the turbulent and wave regions. A short discussion and conclusions drawn from the present study are given in $\S 5$. 


\section{The numerical method and initial condition}

The numerical simulation to be analyzed in the present paper used the channel flow code developed by Kim, Moin \& Moser (1987). It was originally developed for simulation of fully developed turbulence and uses discretizations in Fourier modes in the streamwise $(x)$ and the spanwise $(z)$ directions. In the normal (to the wall) direction $(y)$, the flow quantities are expanded in Chebychev polynomials. The time advancement is done with an implicit Crank-Nicolson scheme for the viscous term and with an explicit Adams-Bashforth scheme for the nonlinear terms. The present simulation used a computational domain of $35 \pi \times 2 \times 25 \pi$ in the streamwise, normal, and spanwise directions, respectively. All quantities are normalized by using appropriate combinations of the channel half-height, $h$, and the undisturbed centerline velocity, $U_{C L}$, unless otherwise stated. The domain was discretized using $256 \times 33 \times 256$ spectral modes in the respective directions. In the spanwise direction only half the number of modes were actually used since symmetry was assumed in that direction. The aliasing errors associated with computing the nonlinear terms pseudo-spectrally were removed by the $3 / 2$-rule, i.e., the collocation points used in physical space were 1.5 times the number of spectral modes in the streamwise and spanwise directions.

The Reynolds number $\left(R e=U_{C L} h / \nu\right)$ was chosen to be 1500 , the same as that of the experiments of Henningson \& Alfredsson (1987), and the pressure gradient was kept constant during the simulation. The spot was initialized using a velocity field consisting of a vortex pair, which had the following analytical form

$$
\begin{aligned}
\psi & =\left(1-y^{2}\right)^{2} z e^{-16 x^{2}-4 z^{2}} \\
u & =0 \\
v & =\psi_{z} \\
w & =-\psi_{y}
\end{aligned}
$$

where $u, v$, and $w$ are the streamwise, normal, and spanwise velocity components, respectively. This simple double vortex was adapted from the initial disturbance used by Henningson (1988), where its inviscid linear development was considered. A similar disturbance has also been used by Russell \& Landahl (1984) in a model of the turbulent bursting process. The large computational domain was chosen to allow a spot to grow to a self-similar state independent of the initial disturbance. The collocation points (associated with the dealiased grid) were spaced a distance of 0.29 length units apart in the streamwise direction, 0.004-0.09 in the normal direction (the spacing varies according to the Chebychev distribution), and 0.20 in the spanwise direction. Since the Reynolds number was low, this was judged to 
be adequate. In terms of viscous wall units, based on the mean friction velocity inside the turbulent part of the spot, the above spacing corresponds to $20,0.28$ 6.3 , and 15, respectively. This is about a factor of two greater than typical values used in full turbulence simulations (Kim, Moin, \& Moser, 1987). The computed spectra of the modes in the series expansions did not reveal any abnormalities in high wavenumbers, even though the resolution was marginal.

The large amplitude disturbance quickly broke down and developed into a turbulent spot as it propagated downstream. The time step in the simulation was $\Delta t=0.03$ and the calculation was continued up to $t=270$. At this point the spot started to fill up the computational domain. Henningson, Spalart, \& Kim (1987) found that, except for the change of shape of the front region of the spot, the development is self-similar (see fig. 2 in their paper). This property will be exploited when spatial variations of the flow quantities obtained from the simulation are compared to temporal variations obtained from experiments by Klingmann, Alfredsson \& Henningson (1990). Both results are transformed into conical coordinates, i.e. $\xi=$ $x / t$ and $\zeta=z / t$, for comparison. In these coordinates the spot will be approximately stationary. The present investigation will concentrate on the fully developed spot, with most data obtained from the velocity field at $t=258$. This time was chosen because the spot had already acquired all of the characteristics of the experimentally observed spot, but it had not become large enough for substantial interference with neighboring spots (because of the periodicity in the horizontal directions). Thus, unless stated otherwise, the data presented will be taken from velocity fields at $t=258$.

\section{Velocity fields}

In this section instantaneous velocity fields and a suitably defined mean velocity will be analyzed in detail for various regions of the spot.

\subsection{Instantaneous velocity}

Color-coded contours of the instantaneous velocities for all three components at $y=0$ (centerline of the channel) and contours for the streamwise component at $y=0.71$ are shown in figure 1 . Note that only level curves of the velocity fields are plotted. Thus, the part of the plots where steep variations occur will have closely spaced contours, giving a higher intensity in color than the parts of the flow field where spatial variations are slow. In this way the laminar and turbulent regions are easily distinguished. In all of the color figures the streamwise axis extends from left to right and the spanwise from top to bottom, and the spacing between tick marks on the streamwise axis is 10 units. The contours of the streamwise velocity in figure la show a slower moving turbulent part (blue-green-yellow contours), with 
the lowest value found at the spanwise wingtips. Around the turbulent part, the much smoother and sparser contours of the disturbed laminar flow can be seen (the red to pink curves outside the spanwise wingtip). In contrast to the velocity in the turbulent region, this flow is faster than the undisturbed centerline velocity. In front of the spot a disturbed but non turbulent region with an arrowhead shape can be seen. In the arrowhead, where the flow was initially turbulent ${ }^{1}$, the turbulence production is apparently not large enough to sustain a turbulent flow. The normal velocity, seen in figure $1 \mathrm{~b}$, does not show the arrowhead at all, instead it has the typical crescent shape seen in flow-visualization pictures. The normal velocity also shows the characteristic large amplitude wave packet at the spanwise wingtips. Notice, by the appearance of the red and dark blue colors, that the waves increase drastically in amplitude the closer they are to the fully turbulent region. Note also, apart from the wave packet, that the normal velocity has large fluctuations only in the turbulent region, making it suitable as a marker of the interface between laminar and turbulent flow. Contours of the spanwise velocity, figure 1c, show a mean motion out towards the wingtips in the turbulent part (yellow color). This outward motion is largest at the wingtip, as indicated by the green and blue curves. This outward motion continues in the laminar flow outside the wingtip, whereas there is a motion towards the spot downstream of the turbulent part (red color). Combining the features of the streamwise and spanwise velocities, we find that the slower moving turbulent region acts as a partial flow blockage, causing the laminar fluid to accelerate around the spot. The idea of blockage was originally proposed by Widnall (1984) in an early attempt to model the Poiseuille spot.

Figure 1d shows contours of the streamwise velocity closer to the wall. In the turbulent part the typical low- and high-speed streaky structures usually associated with turbulent flows can be seen. The velocity of the undisturbed flow at this $y$-location is 0.5 , which is represented with a green color (seen at a few positions between the blue and yellow). Most of the disturbances in the turbulent region are color-coded yellow to red, indicating a higher velocity than the undisturbed region. Thus, in contrast to the flow at the centerline, the turbulent flow closer to the wall in general has a higher velocity than the surrounding laminar flow. Note also that the waves at the wingtips can be seen in the contours of the streamwise velocity at this $y$-location. Since these waves consist of the least stable Orr-Sommerfeld mode (Henningson, 1989), they have a symmetric transverse distribution for the normal velocity and an anti-symmetric transverse dependence for the streamwise and spanwise velocities. Thus, the waves can be seen only in the normal velocity at the centerline:

1 The shape of the spot for early times show an arrowhead shape in the downstream direction (Alavyoon, et al., 1987). 


\subsection{Averaged velocities}

Because of the substantial computational effort required, no attempt was made to run several cases to obtain ensemble-averaged statistics. In order to compare the simulation results from a single realization to the ensemble-averaged results obtained from experiments, a mean velocity has to be defined. Analysis of the fluctuating components also requires a properly defined mean. Since the spot is approximately self-similar, the conical averaging procedure was tried first. One hundred and twenty velocity fields between $t=222$ and $t=258$ were interpolated into the horizontal conical coordinate system and averaged. The velocity obtained from this procedure was not smooth enough to be regarded as a mean. The conicalaveraged velocity field still contained a large number of small-scale structures that survived the averaging process.

Several different spatial averages were tried next, with the best results obtained by use of Gaussian filters. The mean was defined in the following way,

$$
\begin{aligned}
\langle u(x, y, z)\rangle & =\frac{1}{\pi \sqrt{a b}} \iint f\left(x-x^{\prime}, z-z^{\prime}\right) u\left(x^{\prime}, y, z^{\prime}\right) d x^{\prime} d z^{\prime} \\
f(x, z) & =\exp \left(-(x / a)^{2}-(z / b)^{2}\right)
\end{aligned}
$$

Here \langle\rangle denotes the average obtained by applying the Gaussian filter. The above convolution was transformed into Fourier space, where it becomes a simple multiplication

$$
\langle\hat{u}(\alpha, y, \beta)\rangle=\hat{f}(\alpha, \beta) \hat{u}(\alpha, y, \beta)
$$

where $\alpha$ and $\beta$ are the streamwise and spanwise wavenumber, respectively, and the caret signifies a Fourier transformed quantity. The filter widths were determined such that the filtering process removed small- and medium-scale structures in the turbulent region without altering the largest scales of the spot. In our case we chose $a=3.5$ and $b=2.5$. To get an idea of the effect of this filter, compare figure $3 \mathrm{a}$ and figure $8 \mathrm{a}$, where the Gaussian-averaged velocity and the corresponding disturbance velocity can be seen. Notice that all of the small scales usually associated with the turbulent fluctuations are contained in the disturbance field, while the mean motion is smooth (see also discussion in $\S 4$ ).

In figures 2 and 3a, an ensemble-averaged field from Klingmann, Alfredsson, \& Henningson (1990) and that obtained from the Gaussian-averaged velocity field are shown. Agreement between the two results is remarkable. Note, for instance, the sharp edge region with the dip in the velocity, the arrowhead front part of the 
disturbance, and the excess laminar velocity around the spot: The agreement is not only qualitatively good, but corresponds for the most part also quantitatively. Averages at other $y$-positions show similar agreement. Although they are not shown here, it should be noted that averages from the experiments and the simulation at earlier times do not agree, because of differences in initial conditions.

The Gaussian-averaged velocity field chosen as the representation of the mean velocity is shown in figures $3 \mathrm{a}$ through $3 \mathrm{~d}$. These velocity fields show many of the same features as the instantaneous fields already discussed. The main characteristics of the mean velocities at the centerline (figs. $3 a$ and $3 b$ ) are: the lower streamwise mean inside the turbulent region and the faster motion outside; the outward spanwise mean inside the spot, continuing well into the laminar region; and the flow towards the spot downstream of the turbulent area. Closer to the wall the the mean streamwise velocity in the turbulent region is higher than the surrounding laminar flow (fig. 3c). At the wingtip there is now a maximum rather than the minimum seen at the centerline. In the laminar region outside the wingtip, however, the streamwise velocity is still larger than the undisturbed value. In contrast, the spanwise velocity closer to the wall, figure $3 \mathrm{~d}$, exhibits the same features as it does at the centerline. The outward motion as well as the region of flow towards the spot, however, have a lower amplitude. These features have also moved considerably further upstream, indicating that the disturbed region is inclined.

To study the motion of the fluid at the spot interface, the velocity of the fluid relative to the velocity of the interface was investigated. The spot interface was defined as a smooth envelope around the region where the normal velocity exceeded \pm 0.02 . As noted previously, the appearance of normal velocity serves as a good marker for the edge of the spot. ${ }^{2}$ The streamwise velocity of the interface was then assumed to vary linearly between the rear and leading edges, as was the spanwise velocity between the symmetry line and the wingtip region. Since the conical similarity (in $x$ and $z$ ) of the overall spot features is well established (see Henningson, Spalart, \& Kim, 1987), this procedure can be used with confidence. Note that the front part, where the conical similarity does not apply, will not be analyzed with this technique. The interface velocity was subtracted from the local mean fluid velocity at the spot edge and the result was decomposed into components parallel and perpendicular to the spot edge (fig. 4). At the centerline, figure $4 \mathrm{a}$, the component perpendicular to the interface indicates a fluid motion into the spot at the back and out of the spot at

2 Here it is not possible to use the deviation of the streamwise velocity from its laminar value to define the spot edge, as has usually been done in boundary-layer spots. Because of the blockage effect, the horizontal velocity components are disturbed well into the laminar region in the Poiseuille case. 
the front. At the wingtip, however, the component perpendicular to the interface velocity is about the same as the fluid velocity in that direction. Thus, in the mean no fluid is crossing the interface at that position. The component of the fluid velocity parallel to the spot edge is larger than the interface velocity at all positions and represents a flow around the spot. Closer to the walls, figure $4 \mathrm{~b}$, the relative motion is different. In the front part of the spot, the fluid velocity perpendicular to the spot edge indicates a flow into the spot, and the component parallel to the edge indicates a flow around the spot in the upstream direction. In the rear part of the spot, however, there is almost no relative motion between the fluid and interface at this $y$-location. Note that at positions even closer to the wall the fluid velocity is directed out of the spot at the rear interface. Thus, except for a few positions, the spot edge is not a material boundary, but fluid particles are moving in and out of the turbulent region. This suggests that not only is nonturbulent fluid entrained into the spot, but turbulent fluid may also be ejected out of the spot and subsequently relaminarized. A comparison of the position of the spot edge at the two $y$ locations shown in figure 4 shows that it shifts upstream as one moves closer to the walls.

\subsection{Characteristics of the wingtip region}

The large-amplitude wave packet at the wingtips is one of the most interesting characteristics of the turbulent spot in plane Poiseuille flow. The spatial variation of the waves in a spanwise cut in the center of the channel is shown in figure 5a. Within a few wave lengths the amplitude can be seen to grow an order of magnitude. A cause for the growth can be found by examining the mean velocity profiles at the position of the waves. Figures $5 \mathrm{~b}$ and $5 \mathrm{c}$ show the streamwise and spanwise mean components in that area. The streamwise velocity profile changes from a typical laminar Poiseuille-like profile to a more flat, turbulent-like profile inside the spot. The spanwise velocity, on the other hand, can be seen to have inflexion points. These inflexional spanwise profiles are potentially unstable to oblique waves much in the same manner as crossflow instability exists in threedimensional boundary layers. Stability calculations by Henningson (1989), using mean profiles obtained from the present simulation, showed that this is indeed the case and that amplification rates are large enough to explain most of the growth observed in the simulation. An additional mechanism for growth of the waves in the transition area was also found. It resulted from the inhomogeneity of the background mean, which caused convergence of wave energy and thus an increase in the amplitude of the waves. This wave-energy focusing mechanism was originally proposed by Landahl (1972) as a mechanism for wave breakdown. The reader is referred to Henningson (1989) for further details regarding the wave analysis.

The breakdown of the waves is further illustrated in figure 6 . Here \pm 0.01 contours 
of the normal velocity are plotted for five closely spaced times. This gives us the possibility to study the propagation and breakdown characteristics of the waves. Allowing for the variation in the wave group, the phase velocity (in the direction of the wavenumber vector) was found to be -0.23 with a wave angle of $-68^{\circ}$. The group velocity is harder to estimate since the waves can grow only on the inhomogeneous laminar flow outside the turbulent part, and thus are trapped in that region. An estimate of the group velocity using linear stability theory gives values around 0.6 in the streamwise direction and -0.1 in the spanwise direction. In figure 6 one may follow the waves from their birth outside the wingtip area to their eventual breakdown inside the turbulent region. If we follow the outermost wave trough in figure $6 \mathrm{a}$, it can be seen in the subsequent pictures to move toward the turbulent region: A new wave crest is meanwhile appearing outside the trough. If we instead follow the second outermost wave crest in figure $6 \mathrm{a}$, it can be seen to have broken down completely by figure 6e. This clearly illustrates that, in a frame of reference moving with the spot, the waves move into the spot where they break down into smaller-scale motion. Figure 7 shows a blow up of the wave region for a time close to that of figure $6 \mathrm{~d}$.

\section{Characteristics of the turbulent part}

By subtracting the spatially averaged mean from the instantaneous velocity, we obtain a fluctuating disturbance velocity, $u^{\prime}=u-\langle u\rangle$. The streamwise disturbance velocity at $y=0.83$ is shown in figure $8 \mathrm{a}$. The small-scale features are essentially the same as the instantaneous velocity field shown in figure $1 \mathrm{~d}$. The streaky structures are even more pronounced, with some streaks going through half the length of the spot. The waves at the wingtip can also be clearly seen. This confirms that the width of the spatial filter is wide enough to average out any fluctuations associated with the small-scale turbulence, leaving them in the disturbance fields. From the fluctuating components the r.m.s. values can be calculated using the same Gaussian filter used to define the mean velocities, e.g., $u_{r . m . s}=\sqrt{\left\langle(u-\langle u\rangle)^{2}\right\rangle}$. The r.m.s. fluctuation of the normal component at $y=0$ (fig. $8 \mathrm{~b}$ ) rises to twice its average value.in the wingtip region. This is a consequence of the high-amplitude wave packet present there. Thus, it is also clear that the turbulence statistics would reflect the difference in the behavior of the spot in the region where waves are found, compared to the area where there are no waves. Instead of averaging these regions together when overall statistics were computed, the averaging was done separately in the two regions. An area inside the spot defined by $0.58<\xi<0.70$ and $-0.08<\zeta<0.0$, will be referred to as the turbulent area, while an area including $0.70<\xi<0.74$ and $-0.12<\zeta<-0.08$ will be referred to as the wave area (fig. $8 b$ ).

\subsection{Turbulence statistics}


In order to compare turbulence characteristics inside the spot with those in fully developed channel flow turbulence, inner scaling was used, i.e., all quantities are normalized with the friction velocity $u_{\tau}=\sqrt{\nu d\langle u\rangle /\left.d y\right|_{\text {wall }}}$ and the kinematic viscosity $\nu$. A mean value for $u_{r} / U_{C L}$ of 0.05 inside the spot was obtained by averaging it in the turbulent area. The $y$-dependence of the mean streamwise velocity in the turbulent area is shown in figure $9 \mathrm{a}$. The log-region is very small for the present case because of the low Reynolds number $\left(R e_{\tau}=u_{\tau} h / \nu \approx 70\right.$; note that the undisturbed laminar value is about 50). Considering the low Reynolds number the mean flow has the characteristics of a fully developed turbulent channel flow, i.e., the linear region close to the wall and the (small) log region away from the wall. The distribution of the r.m.s. values of the three velocity components averaged over the turbulent area are shown in figure $9 \mathrm{~b}$. When they are compared to the same quantities in the wave area, figure $9 \mathrm{c}$, it is apparent that all the fluctuations are higher in the wave area. The normal velocity is particularly high and has a different shape as well. In figure 9d the Reynolds shear stress in the turbulent and wave area are shown. Again, the magnitude in the wave area is much higher than in the turbulent area, indicating that the momentum transfer from the mean to the disturbance velocities is high in the wave area. Although a large part of the Reynolds stress in the wave area can be expected to consist of contributions from the waves before they have broken down, the fact that they do break down and add to the turbulent part of the spot (c.f. §3.3) suggests that one may also consider their part of the Reynolds stress as turbulent production. The salient feature of the Reynolds shear stress in the turbulent area is the fact that it is close to a straight line (with slope -1) in the center of the channel, indicating that the turbulence inside the spot has reached a statistically steady state.

In figures 10a,b,c the r.m.s. values and the Reynolds stress from the turbulent area are compared with those in a fully developed turbulent channel flow, (Kim, Moin, \& Moser, 1987). Figures 10a,b are scaled with wall units and show agreement between the two cases close to the wall. Further out the curves separate because of the difference in Reynolds number. The Reynolds number based on the friction velocity and channel half-height was 180 in the simulation of Kim, Moin, \& Moser (1987) and 70 in the present one. Figure 10c shows that agreement in the center of the channel can be obtained if the normal coordinate is scaled with the channel half-height rather than with wall units. The overall comparison indicates that the fluctuating quantities in the turbulent part of the spot behave as if they were part of a fully developed turbulent flow. The somewhat low value of the peak in the streamwise r.m.s. velocity can be attributed to the low Reynolds number in the present investigation. Note that Spalart (1988) found that for the boundary layer the peak of the streamwise r.m.s. velocity decreased for low Reynolds number. 


\subsection{Turbulence structure}

We have already discussed the existence of the streaky structures in the near-wall region in the turbulent area. Another characteristic of wall-bounded turbulent shear flows is the sharp shear-layer structures found in the near-wall region. The low-speed streaks found close to the walls are seen to lift away from the wall quasi-periodically. The main effect of the lift-up of the streaks is to create shear layers. These shear layers have been found to be the major contributor to the turbulent production (Johansson, Alfredsson, \& Kim, 1990). One frequently used approach to detect the shear layers is to locate them with the VISA (Variable-Interval-Space-Average) technique. This is the spatial counterpart of the VITA technique which uses a time rather than a space average, (Blackwelder \& Kaplan, 1976). Kim (1983) found that the two approaches were equivalent in term of the turbulence structure selected. An event is considered to have occurred when the local streamwise variance exceeds a threshold, $k$, times the mean streamwise r.m.s. value, i.e.,

$$
\frac{1}{L} \int_{-L / 2}^{L / 2} u^{2}(x-s) d s-\left(\frac{1}{L} \int_{-L / 2}^{L / 2} u(x-s) d s\right)^{2}>k u_{r . m . s .}^{2}
$$

$L$ is the averaging length, which for our case was about 200 viscous length units, and $k$ was chosen to be 0.8 . Islands of high variance of streamwise velocity were identified at $y^{+}=12$ at both walls. In figure 11 typical islands obtained with this technique are shown. They can be seen to be distributed intermittently in the turbulent area, in addition to a cluster in the wave region. An ensemble averaged velocity field of a typical event was obtained by a sum of their individual velocity fields. Again, when averages are made we must distinguish between the wave and turbulent regions. In all events the maximum local variance in the associated islands was determined and used to align the averages. The results obtained using this procedure on all events detected in the turbulent area are given in figure 12, where the mean turbulent velocity has been subtracted from the streamwise component. Figure 12a shows the streamwise velocity component, with the typical inclined shear-layer structure associated with the lift-up of low-speed fluid at the downstream side, and the downward sweep of high-speed fluid at the upstream side of the shear layer. The horizontal structure of the shear layer is shown in figure $12 \mathrm{~b}$. The large-amplitude central lobes correspond to the shear layer seen in the previous figure. Outside the central downstream negative lobe are two smaller positive ones, representing a higher streamwise velocity than the mean. On the upstream half of the shear layer, the inverted structure can be seen. Because of the relation to the streaks; the spanwise distance between the outer lobes is a measure of the streak width. In our case they are found to be slightly above 100 viscous units apart 
(recall that a half channel height is about 70 viscous units), indicating that the average spacing between the low- and high-speed streaks are the same as that in a fully developed channel flow (Kim, Moin, \& Moser, 1987). The corresponding shearlayer structure obtained by the VISA technique applied to fully developed turbulent channel flow can be seen in figure 13 (from Johansson, Alfredsson, \& Kim, 1990). Note the agreement between the VISA educed shear layers in the turbulent area of the Poiseuille spot and those found in fully developed channel flow.

The ensemble average in the wave area shows a similar shear-layer structure in the $(x, y)$-plane, figure 14a, but the horizontal structure clearly reflects the wavelike characteristics, see figures $14 \mathrm{~b}$ and c. The oblique character of the waves is apparent, in both the streamwise and the normal components. In the cross-wave direction, i.e. the direction perpendicular to the wavenumber vector of the primary waves, there are peak and valley structures. These structures are most pronounced in the normal velocity component and can also be identified in the instantaneous normal velocity field shown in figure 7 and figure $1 \mathrm{~b}$. Similar peaks and valleys in the cross-wave direction ${ }^{3}$ are typicaly seen in vibrating ribbon experiments of the secondary instability process (Klebanof et al. 1962, and Kovasznay, Komoda, \& Vasudeva 1962). In that contex they have been shown to appear as a result of an instability of the elliptical flow in the vortex cores of the corresponding OrrSommerfeld waves (Bayly, Orszag \& Herbert, 1988). Thus a plausible conjecture is that the breakdown of the oblique waves at the wingtips of the spot is also due to the elliptical nature of the flow in their vortex cores. This conclusion is also drawn by Malkus and Waleffe (1990) when they discuss the relation between their model of a single elliptical vortex and the waves at the wingtip of the spot.

\section{Discussion and conclusion}

Although the numerical simulation of the turbulent spot in plane Poiseuille flow considered in this paper had a marginal spatial resolution, the characteristics of the spot are in excellent agreement with those obtained from experimental investigations. The general shape, propagation velocities, and spreading angle are well reproduced in the simulation. In addition, the mean velocities obtained using a Gaussian filter are also in good agreement with the ensemble-averaged results measured by Klingmann, Alfredsson, \& Henningson (1990). Thus, we can conclude that the simulated spot accurately represents a turbulent spot in plane Poiseuille flow.

${ }^{3}$ In the vibrating ribbon experiments peaks and valleys were identified both in the mean and the disturbance velocities. In the spot case we have no way of separating out the mean form the disturbance velocity on the scale of the oblique waves. However, the normal velocity was shown to be associated with the disturbance quantities only. 
Another observation that can be made from the comparison between the simulation and experiments is that the fully developed spot seems to be independent of the initial conditions. A comparison between the early stages of development in the two cases does not show the agreement seen in the later stages. These findings agree with other investigators: for instance, Elder (1962) found that a turbulent spot in the Blasius boundary layer is independent of the initial disturbance as long as the disturbance is large enough to produce a spot.

A flow field containing a fully developed spot was investigated in detail. The mean motion in the central part of the channel was found to have the following characteristics. Nonturbulent fluid outside the spot moves around the turbulent part, with a peak in the streamwise velocity at the wingtip. Inside the spot the mean streamwise velocity had a turbulent character with a lower maximum than that in the laminar region, while the spanwise velocity was directed outward. These findings support the idea of Widnall (1984), that the turbulent part of the spot acts as a partial flow blockage for the laminar fluid outside the spot. The mean outward spanwise component inside the Poiseuille spot has also been identified in boundary-layer spots (Wygnanski, Haritonidis, \& Kaplan, 1976). Close to the walls the mean streamwise velocity inside the spot was larger than the surrounding laminar flow, contributing to the typical "fuller" turbulent mean velocity profile. The mean spanwise motion was found to be similar to the central part of the channel, however, it had an upstream shift which resulted in inflexional profiles in the area outside the wingtips.

The detailed velocity field in the wingtip region of the spot exhibits several interesting characteristics. The inflexional spanwise profiles induce an instability of the cross-flow type, which explains the large amplitudes of the oblique wave packet found in the region. As the waves grow and propagate into the spot, they develop localized shear layers similar to those associated with the breakdown of twodimensional T-S waves (Kovasznay, Komoda, \& Vasudeva, 1962). The shear layers are distributed periodically in the cross-wave direction and are associated with the appearance of peaks and valleys in the velocity field. These similarities with the secondary instability process, as well as the elliptical nature of the primary vorticies in the two cases, suggest that the onset of the cross-wave variation in the oblique wavepacket is a result of the same elliptical instability mechanism that governs the onset of three-dimensionality in the vibrating ribbon experiments. It was also found that the Reynolds shear stress in the wingtip area was about twice as large as that in the turbulent region. Thus, the momentum transfer from the mean to the turbulent flow is enhanced by the growth and breakdown of the oblique wave packet. This scenario is quite different from that found in the boundary-layer spot, where the wave packet is trailing upstream of the turbulent region and has been found 
to propagate away from the spot. There is some indication in the study of Glezer, Katz, \& Wygnanski (1989), however, that the waves in the boundary-layer spot may break down, although they do not seem to play the same fundamental role in the spot spreading process as the waves in the Poiseuille spot.

The turbulence characteristics of the flow inside the spot were also examined. The general conclusion is the same for the Poiseuille spot as for the Blasius spot (Johansson, Her, \& Haritonidis, 1987): the interior of the spot behaves as if it were part of a fully developed turbulent flow. The mean profile has the turbulent characteristics with a linear and a (small) logarithmic region. The profiles of the r.m.s. values and the Reynolds shear stress also agree with those found in fully developed channel flow, indicating that the flow inside the spot has reached a statistically steady state. The structures usually found in turbulent flows were also present in the turbulent part of the spot. Close to the wall we have the typical low- and high-speed streaks, which could be seen to have approximately the same spacing found in fully developed turbulent channel flow. The ensemble-averaged structures obtained from the VISA technique also show a good agreement with those observed in fully developed turbulent flows. The shear layer in the $(x, y)$-midplane displays the associated lift-up of low-speed fluid downstream of the structure and the sweep of higher-speed fluid toward the wall upstream of the structure.

\section{Acknowledgment}

The authors would like to thank Henrik Alfredsson for providing the VISA analysis programs and for valuable assistance in their implementation. The authors would also like to thank Arne Johansson, Marten Landahl and Willem Malkus for many fruitful discussions, as well as Philippe Spalart for careful reading of the manuscript. Part of the present work was done during the 1988 Center for Turbulence Research Summer Program at NASA Ames Research Center. The first author also gratefully acknowledges the support provided by the Swedish Board for Technical Development and the Aeronautical Research Institute of Sweden during the course of this work.

\section{References}

Alavyoon, F., Henningson, D.S., and Alfredsson, P.H. 1986 Turbulent spots in plane Poiseuille flow - flow visualization. Phys. Fluids 29, 1328-1331.

Bayly, B.J., Orszag, S.A. and Herbert, T. 1988 Instability mechanisms in shear-flow transition. Ann. Rev. Fluid Mech. 20, 359-391..

Blackwelder, R.F., and Kaplan, R.E. 1976 On the wall structure of the turbulent boundary layer. J. Fluid Mech. 76, 89-112. 
Carlson; D.R., Widnall, S.E., and Peeters, M.F. 1982 A flow visualization study of transition in plane Poiseuille flow. J. Fluid Mech. 121, 487-505.

Chambers, F.W., and Thomas, A.S.W. 1983 Turbulent spots, wave packets and growth. Phys. Fluids 26; 1160-1162.

Elder, J.W.1962 An experimental investigation of turbulent spots and breakdown to turbulence. J. Fluid Mech. 9, 235-246.

Emmons, H.W. 1951 The laminar-turbulent transition in boundary layer - part I. J. Aero. Sci. 18 490-498.

Gad-el-Hak, M., Blackwelder, R.F., and Riley, J.J. 1981 On the growth of turbulent regions in laminar boundary layers. J. Fluid Mech. 110, 73-95.

Gaster, M. $1975 \mathrm{~A}$ theoretical model of a wave packet in the boundary layer of a flat plate. Proc. Roy. Soc. Lond. A 347, 271-289.

Gaster, M., and Grant, I. 1975 An experimental investigation of the formation and development of a wave packet in a laminar boundary layer. Proc. Roy. Soc. Lond. A 347, 253-269.

Glezer, A., Katz, Y., and Wygnanski, I. 1989 On the breakdown of the wave packet trailing a turbulent spot in a laminar boundary layer. J. Fluid Mech. 198, $1-26$.

Henningson, D.S. 1988 The inviscid initial value problem for a piecewise linear mean flow. Stud. Appl. Math. 78, 31-56.

Henningson, D.S. 1989 Wave growth and spreading of a turbulent spot in plane Poiseuille flow. Phys. Fluids A 1, 1876-1882.

Henningson, D.S., and Alfredsson, P.H. 1987 The wave structure of turbulent spots in plane Poiseuille flow. J. Fluid Mech. 178, 405-421.

Henningson, D.S., Spalart, P., and Kim, J. 1987 Numerical simulations of turbulent spots in plane Poiseuille and boundary layer flows. Phys. Fluids 30, 2914-2917:..

Henningson, D.S., Johansson, A.V., and Lundbladh, A. 1990 On the evolution of localized disturbances in laminar shear flows. To appear in Laminar Turbulent Transition 3, Springer. Proc. of the 3rd IUTAM symposium on laminar turbulent transition, Sep: 11-15, 1989, Toulouse, France.

Herbert, T. 1988 . Secondary instability of boundary layers. Ann. Rev. Fluid Mech. 20, 487-526. 
Johansson, A.V., Alfredsson, P.H., and Kim, J. 1990 Evolution and dynamics of shear-layer structures in near-wall turbulence. Submitted to J. Fluid Mech.; see also Proc. CTR summer program 1987, NASA Ames - Stanford.

Johansson, A.V., Her, J.Y., and Haritonidis, J.H. 1987 On the generation of high-amplitude wall-pressure peaks in turbulent boundary layers and spots. $J$. Fluid Mech. 175, 119-142.

Kim, J. 1983 On the structure of wall bounded turbulent flows. Phys. Fluids26, 2088-2097.

Kim, J., Moin, P., and Moser, R. 1987 Turbulence statistics in fully developed channel flow at low Reynolds number. J. Fluid Mech. 177, 133-166.

Klebanoff, P.S., Tidstrom, K.D., and Sargent, L.M. 1962 The three dimensional nature of boundary layer transition. J. Fluid Mech. 12, 1-34.

Klingmann, B., Alfredsson, P.H., and Henningson, D.S. 1990 An experimental study of the velocity field of turbulent spots in plane Poiseuille flow. Advances in Turbulence 2 (Eds. Fieldler \& Fernholtz), 9-14, Springer.

Kovasznay, L.S.G., Komoda, H., and Vasudeva, B.R. 1962 Detailed flow field in transition. In Proc. the 1962 heat transfer and fluid mechanics institute, Univ. Wash., 1-26.

Landahl, M.T. 1972 Wave mechanics of breakdown. J. Fluid Mech. 56, 775-802.

Landahl, M.T. 1980 A note on an algebraic instability of inviscid parallel shear flows. J. Fluid Mech. 98, 243-251.

Li, F., and Widnall, S.E. 1989 Wave patterns in plane Poiseuille flow created by concentrated disturbances. J. Fluid Mech. 208, 639-656.

Malkus, W.V.R. and Waleffe 1990 The transition from order to disorder in elliptical flow: A direct path to shear flow turbulence. To appear in Advances in turbulence 3 (Eds. A.V. Johansson \& P.H. Alfredsson), Springer.

Maslowe, S.A. 1981 Shear flow instabilities and transition. In Hydrodynamic instabilities and the transition to turbulence (Eds. Swinney and Gollub), 181-228, Springer.

Nishioka, N., Iida, S., and Ichikawa, Y. 1976 An experimental investigation of the stability of plane Poiseuille flow. J. Fluid Mech. 72, 731-751.

Orszag, S.A., and Patera, A.T. 1983 Secondary instability of wall bounded shear flows. J. Fluid Mech. 128, 347-385. 
Russell, J.M., and Landahl, M.T. 1984 The evolution of a flat eddy near a wall in an inviscid shear flow. Phys. Fluids 27, 557-570.

Schubauer, G.B., and Klebanoff, P.S. 1955 Contributions on the mechanics of boundary layer transition. NACA TN-3489.

Spalart, P.R. 1988 Direct simulation of a turbulent boundary layer up to Rtheta=1410. J. Fluid Mech.187, 61-98.

Widnall, S.E. 1984 Growth of turbulent spots in plane Poiseuille flow. In Turbulence and chaotic phenomena in fluids (Ed. T. Tatsumi), 93-98, Elsevier.

Wygnanski, I., Sokolov, M., and Friedman, D. 1976 On a turbulent spot in a laminar boundary layer. J. Fluid Mech. 78, 785-819.

Wygnanski, I., Haritonidis, J.H., and Kaplan, R.E. 1979 On a TollmienSchlichting wave packet produced by a turbulent spot. J. Fluid Mech. 92, 505-528. 


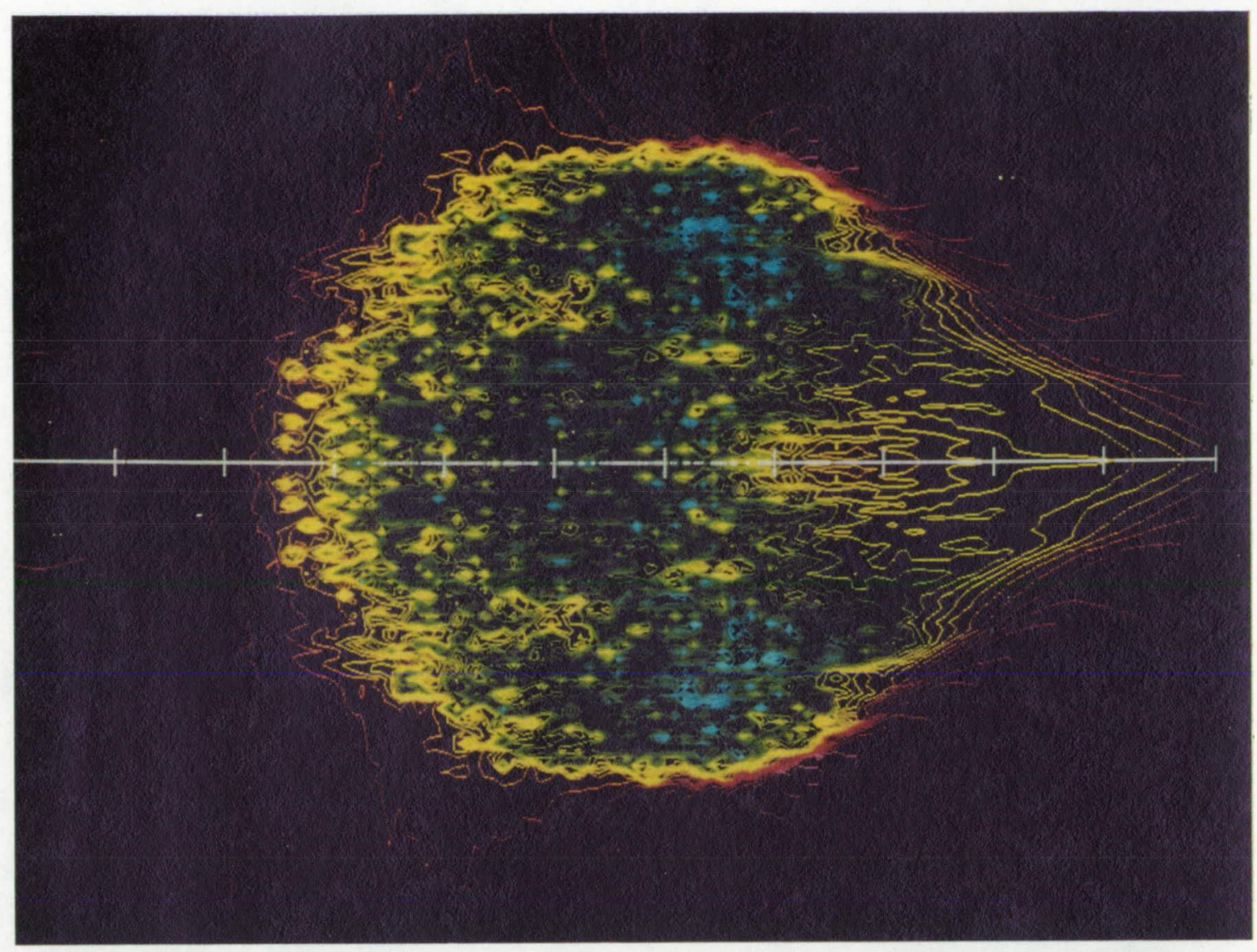

(a)

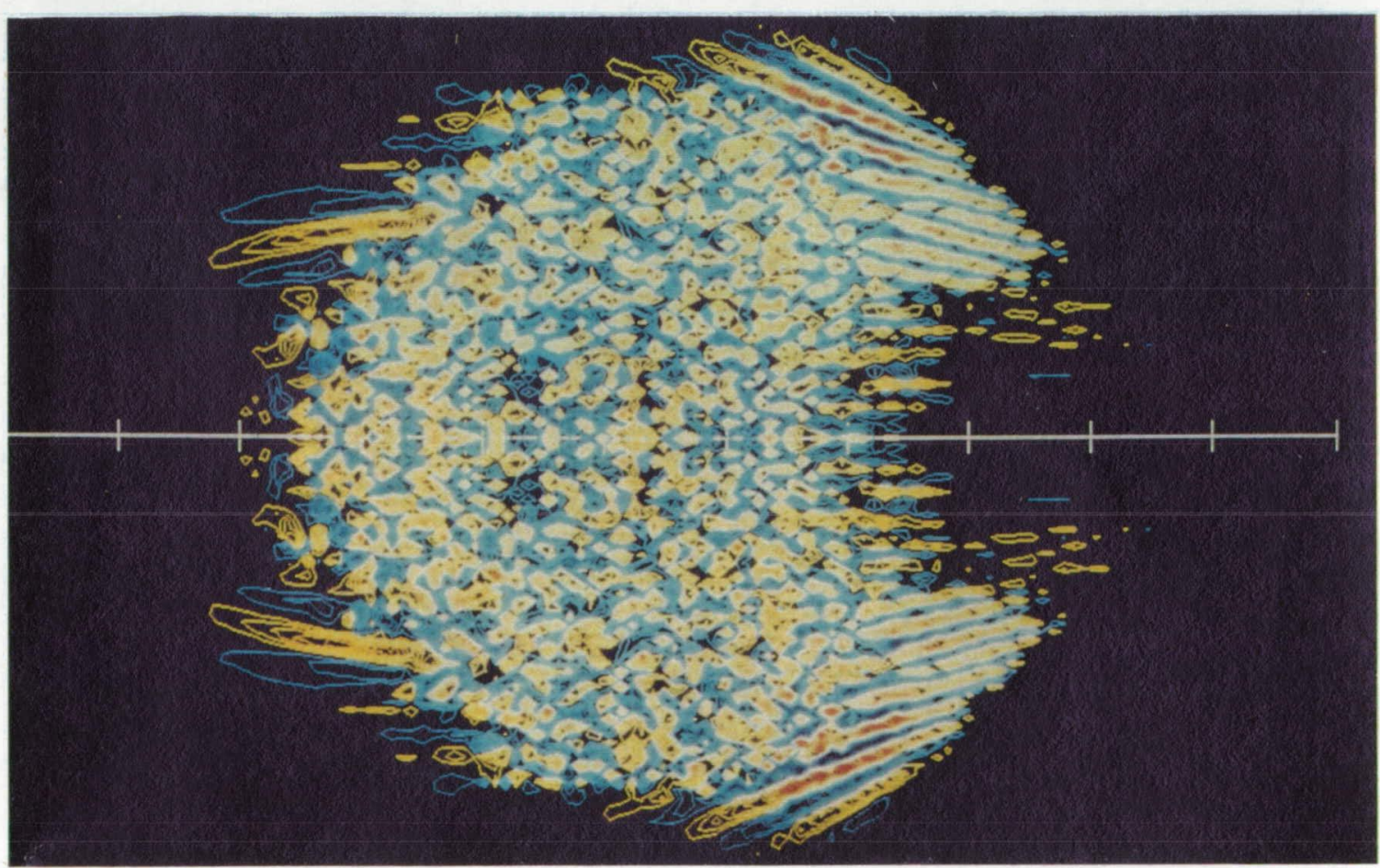

(b)

Figure 1. Contours of instantaneous velocity at $t=258$ of (a) streamwise velocity at $y=0$ (blue, green, yellow, orange, red to pink indicate a velocity range of 0.5 to 1.15 , with orange being about unity); (b) normal velocity at $y=0$ (light to dark blue indicates a velocity range from 0.0 to 0.15 in the negative $y$-direction whereas yellow to red indicates a velocity range from 0.0 to 0.15 in the positive $y$-direction). 


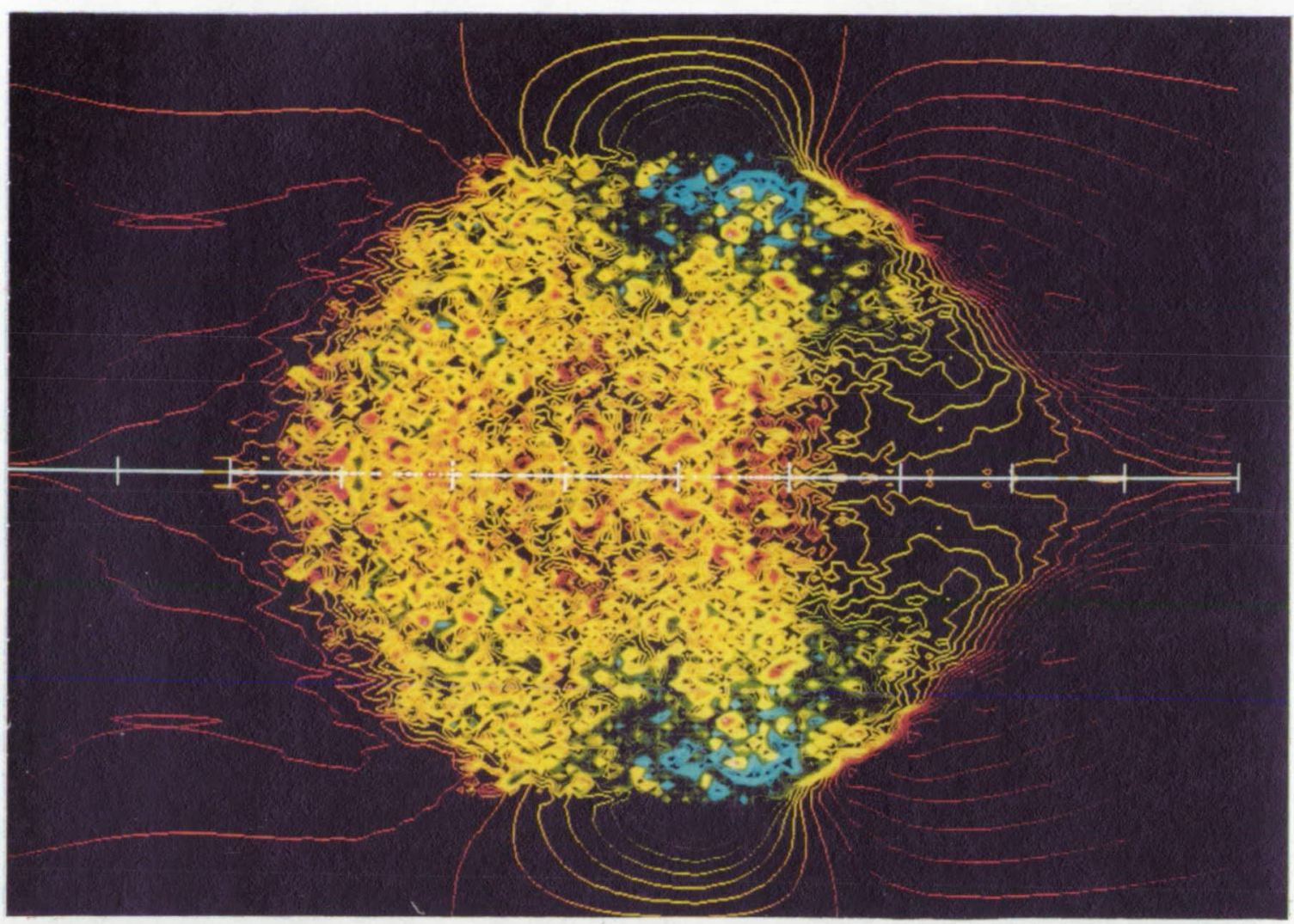

(c)

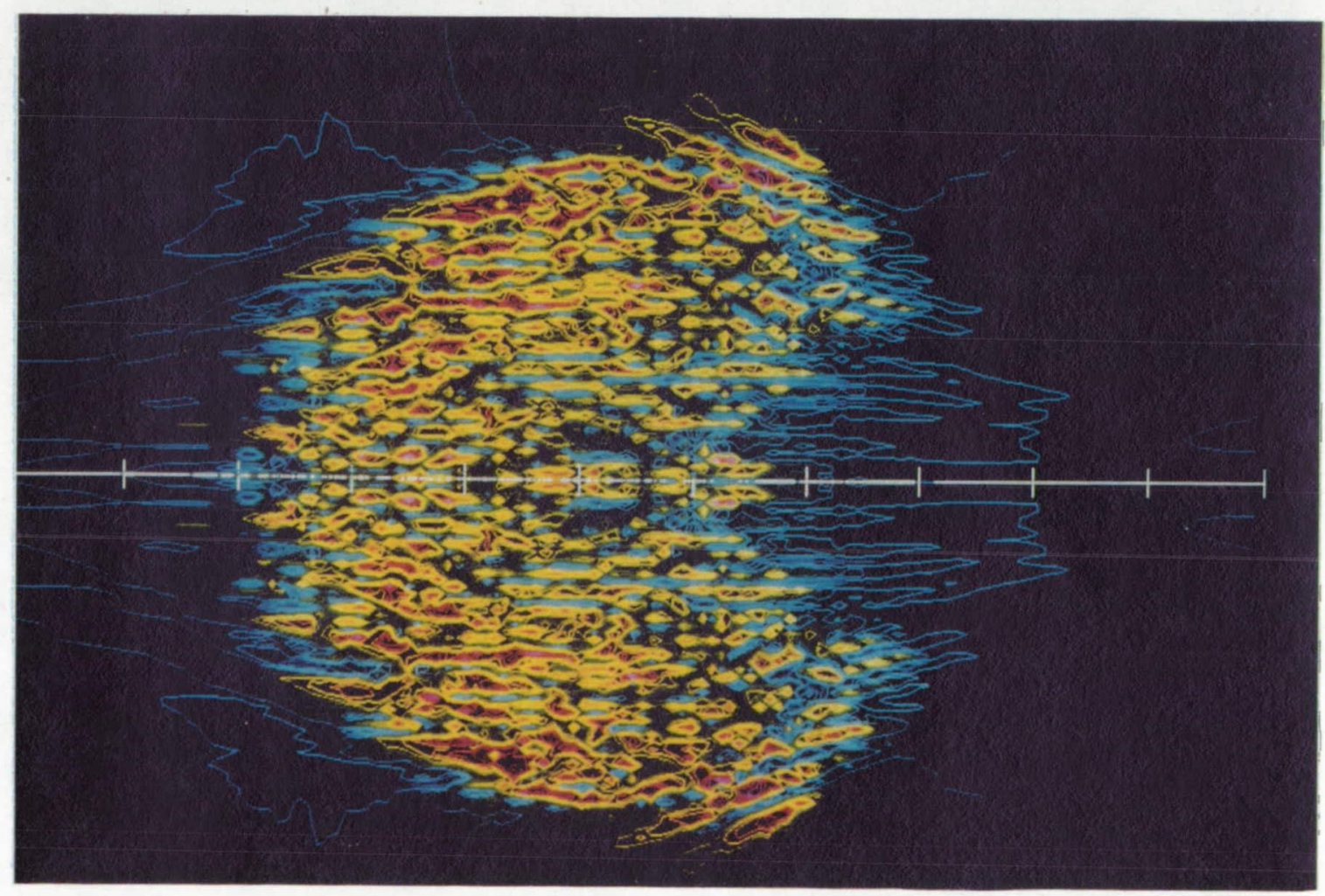

(d)

Figure 1. Concluded. (c) Spanwise velocity at $y=0$ (orange to red indicates a velocity range of 0.0 to 0.06 toward the spanwise symmetry line whereas orange, yellow, green to blue indicate a velocity range of 0.0 to 0.10 away from the spot symmetry line); (d) streamwise velocity at $y=0.707$ (blue, green, yellow, orange to red indicate a velocity range from 0.4 to 0.8 ). The mean flow is from left to right with the streamwise tick marks 10 units apart. 


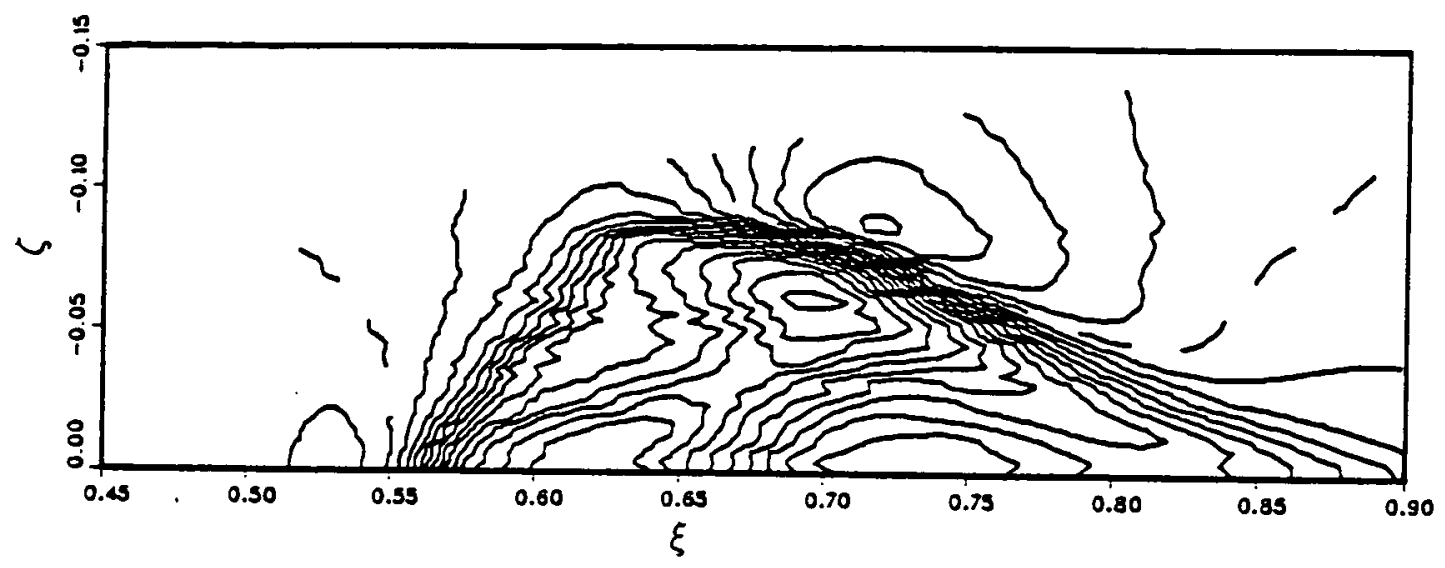

Figure 2. Contours of experimentally ensemble-averaged streamwise velocity at the centerline using 100 different spots at each measurement position. Contour spacing is as in figure 3. 

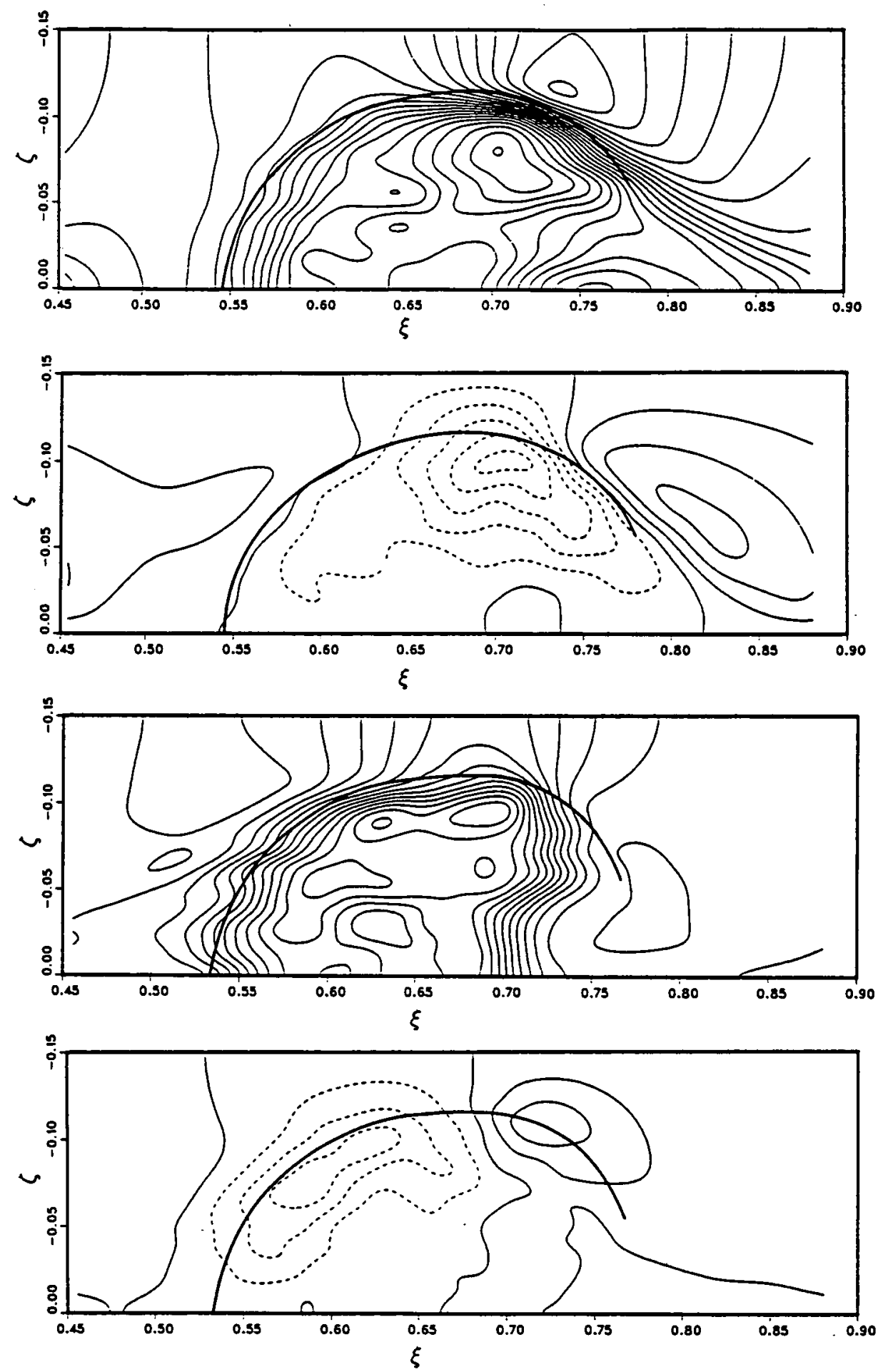

Figure 3. Contours of mean velocities. (a) Streamwise mean velocity at the centerline, obtained using the Gaussian average defined in equation 2. (b) Spanwise mean velocity at the centerline. (c) Streamwise mean velocity at $y=0.83$. (d) Spanwise mean velocity at $y=0.83$. Contour spacing is 0.02 in all cases, with the dashed lines indicating negative contours and the thick solid lines indicating the spot edge. 

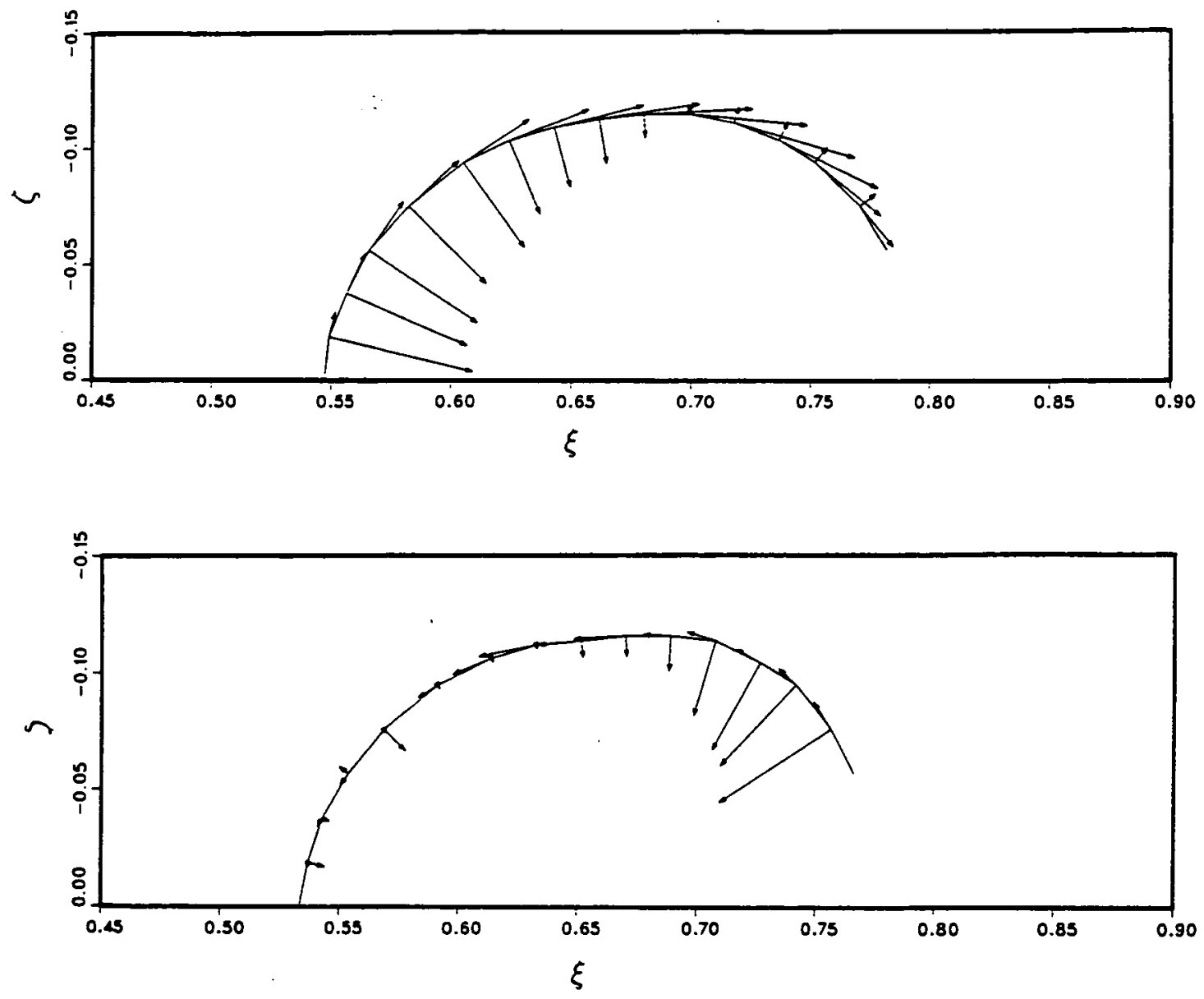

Figure 4. Relative fluid velocity with respect to the spot-interface velocity. Vectors indicate the components of velocity parallel and perpendicular to the edge. (a) $y=0$. (b) $y=0.71$. (The longest velocity vector in (a) represents 0.4 velocity units). 

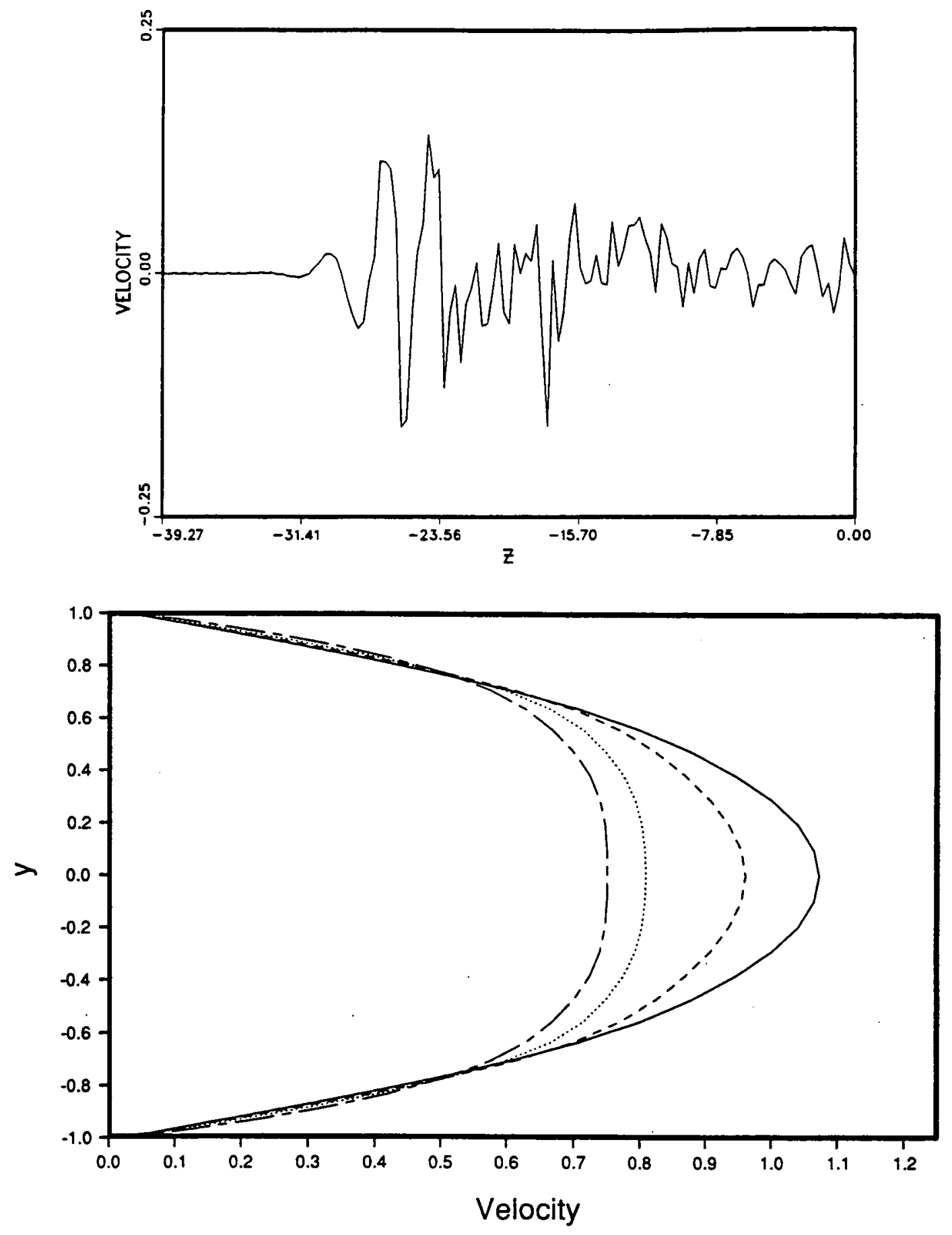

Figure 5. Velocities at the wingtip region. (a) Instantaneous normal velocity at $x=186.0$ and $y=0$. (b) Streamwise mean velocity at $x=186.0$ and $z=-28.8$ (solid), -27.3 (dashed), -25.8 (dotted), and -24.2 (chain dashed). 


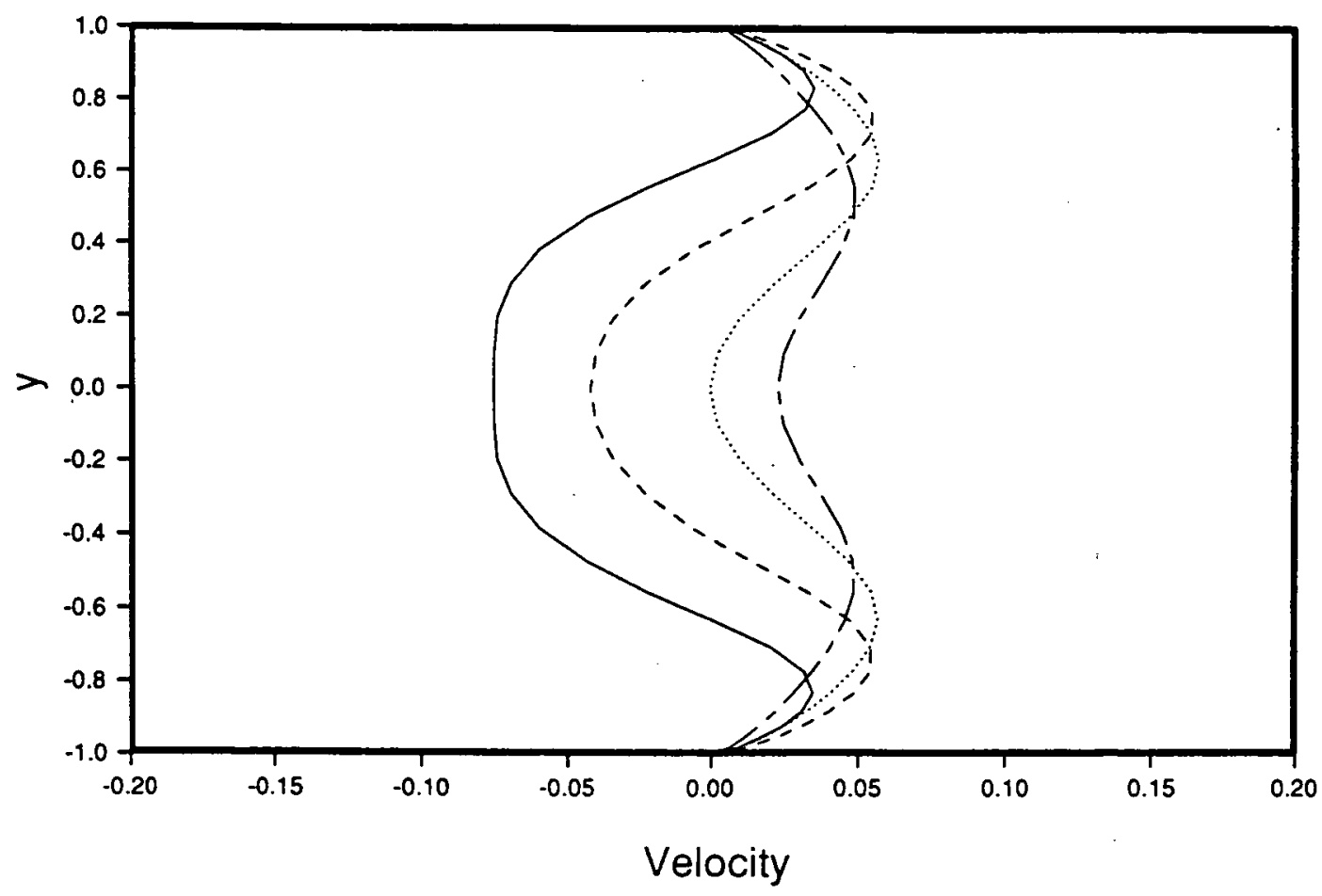

(c) Spanwise mean velocity at $z=-30.4$ for $x=181.7$ (solid), 186.0 (dashed), 190.3 (dotted), and 194.6 (chain dashed). 

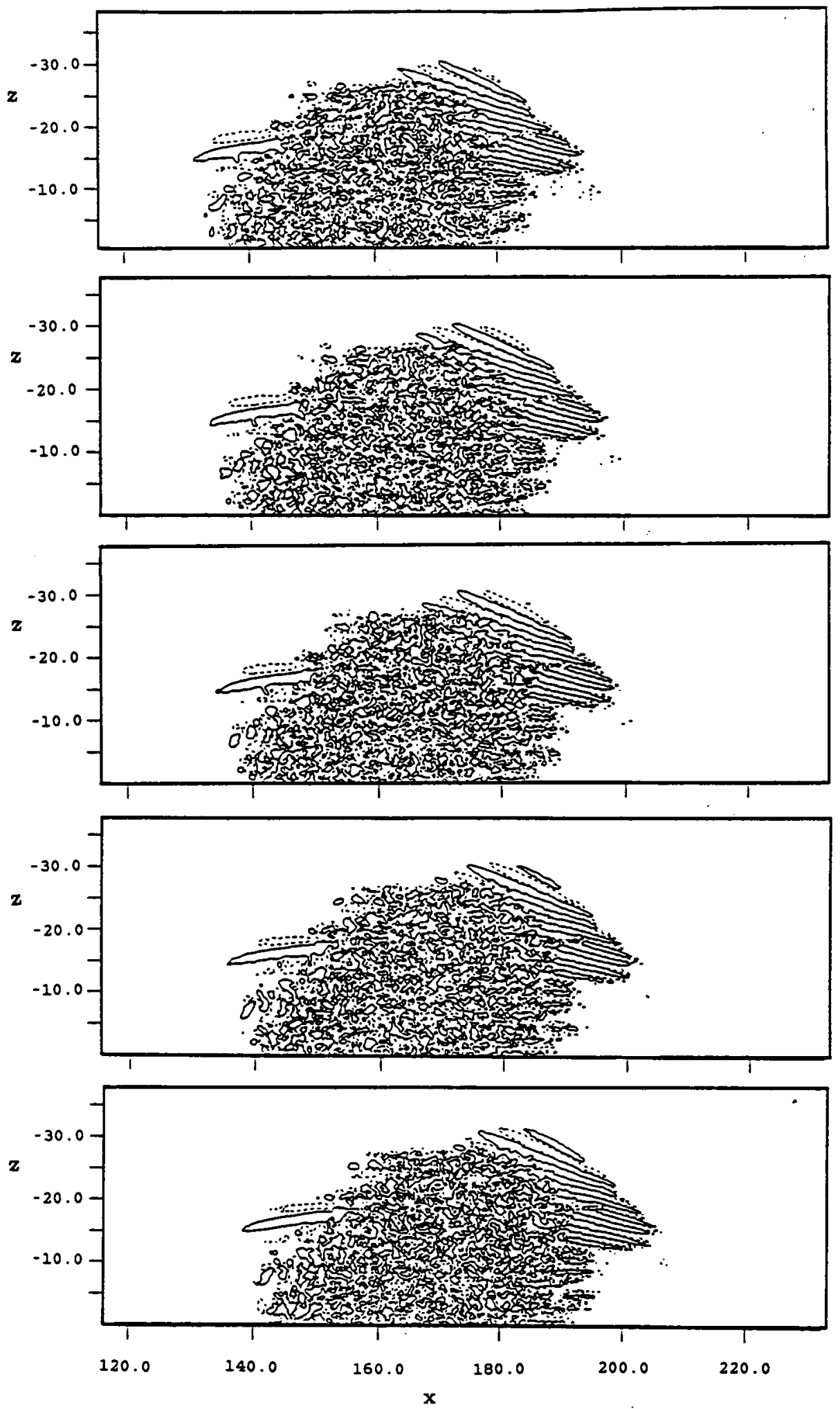

igure 6. Time development of the instantaneous normal velocity at the centerline, $=0.01$ contours at (a) $t=246$, (b) $t=249$, (c) $t=252$, (d) $t=255$, (e) $t=258$. 

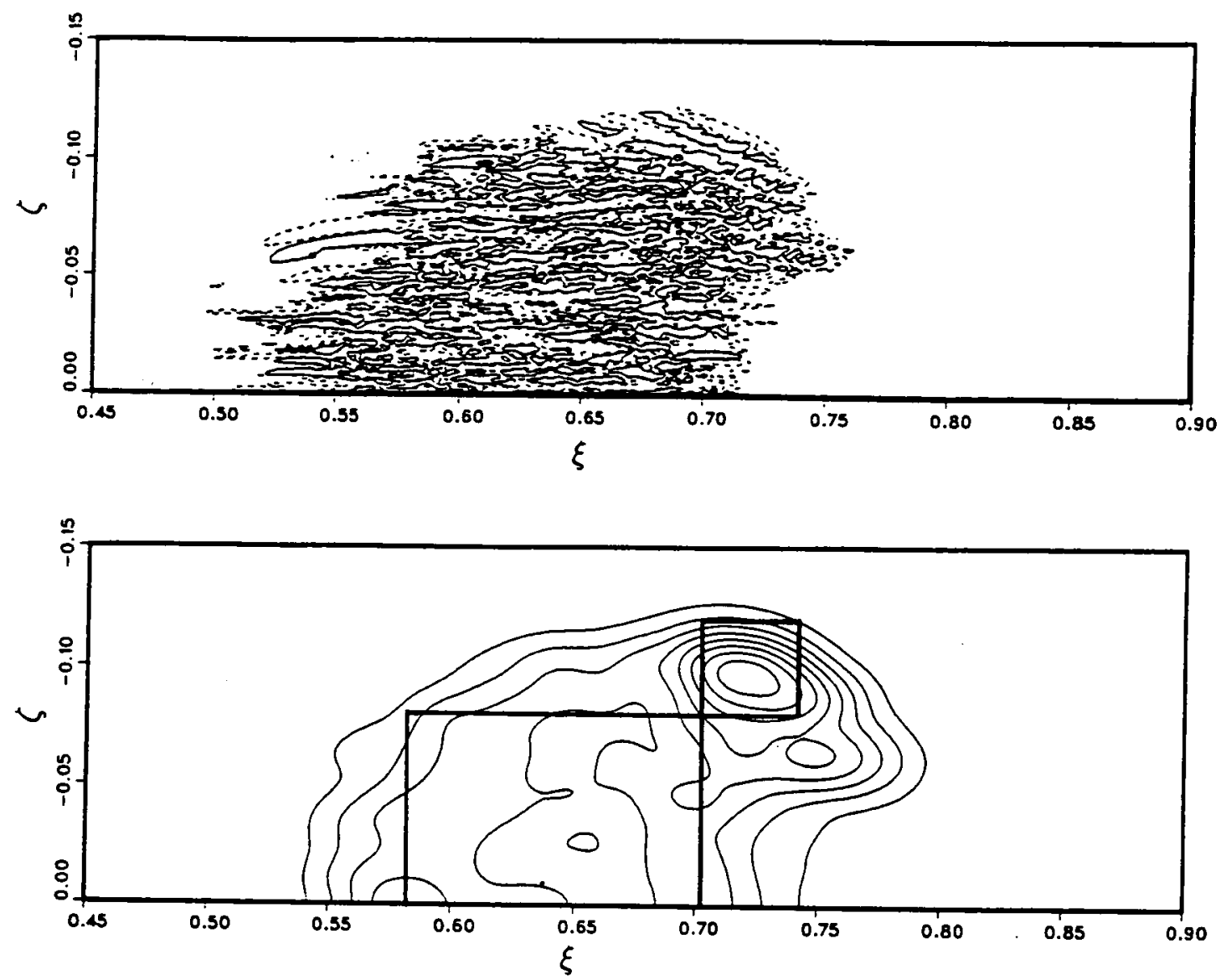

Figure 7. (a) \pm 0.05 contours of the streamwise disturbance velocity at $y=0.83$. (b) Contours of normal r.m.s. velocity at the centerline. Contour spacing is 0.01 , with the outermost contour representing the 0.01 level. The rectangles mark the turbulent and the wingtip area discussed in $\S 4$. 

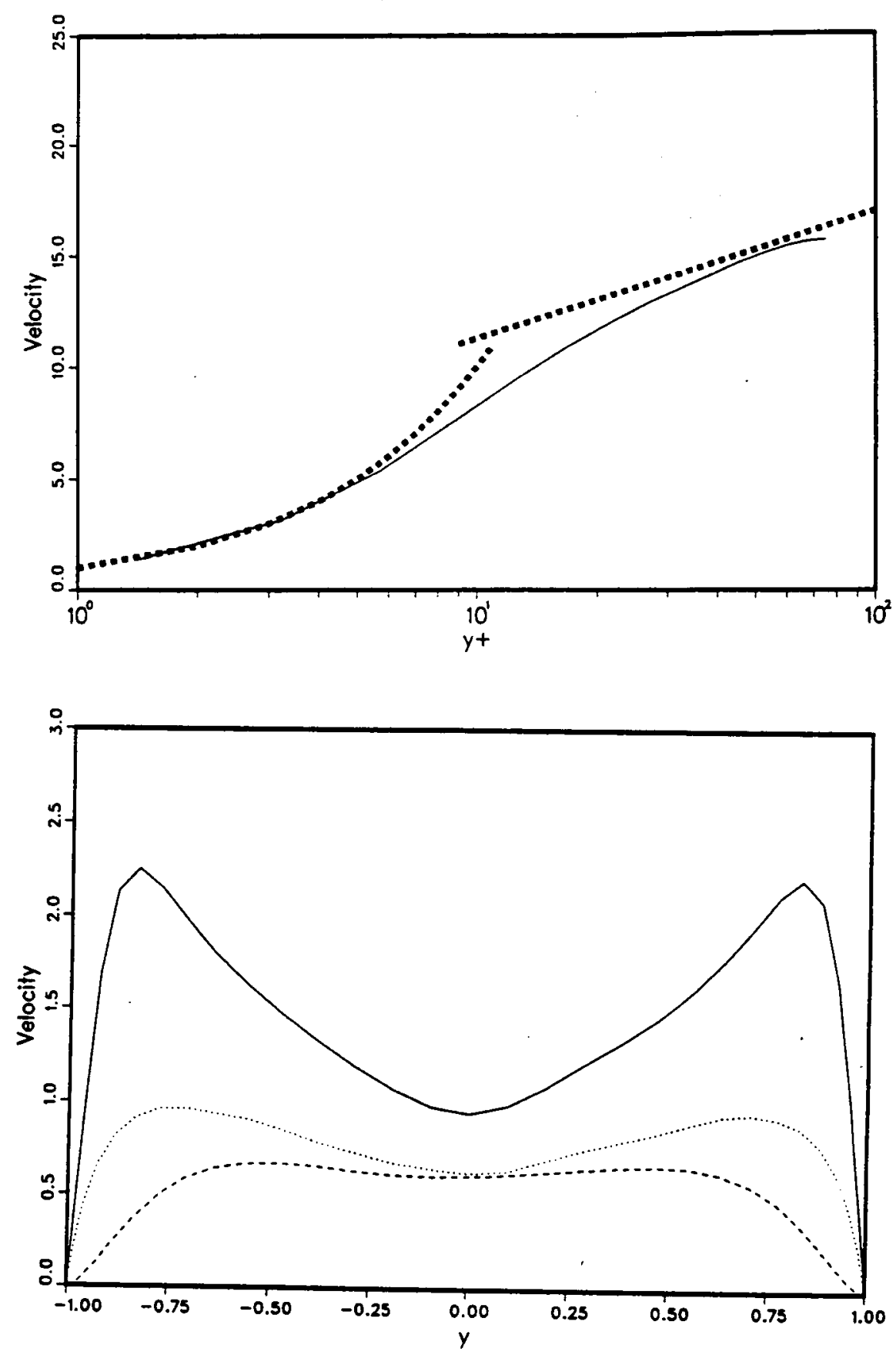

Figure 8. Normal dependence of turbulence quantities plotted in viscous wall units. (a) Mean velocity in the turbulent region obtained from the lower half of the channel; dashed curve is $u^{+}=y^{+}$and $u^{+}=2.5 \ln y^{+}+5.5$. (b) r.m.s. velocities averaged over the turbulent part, 

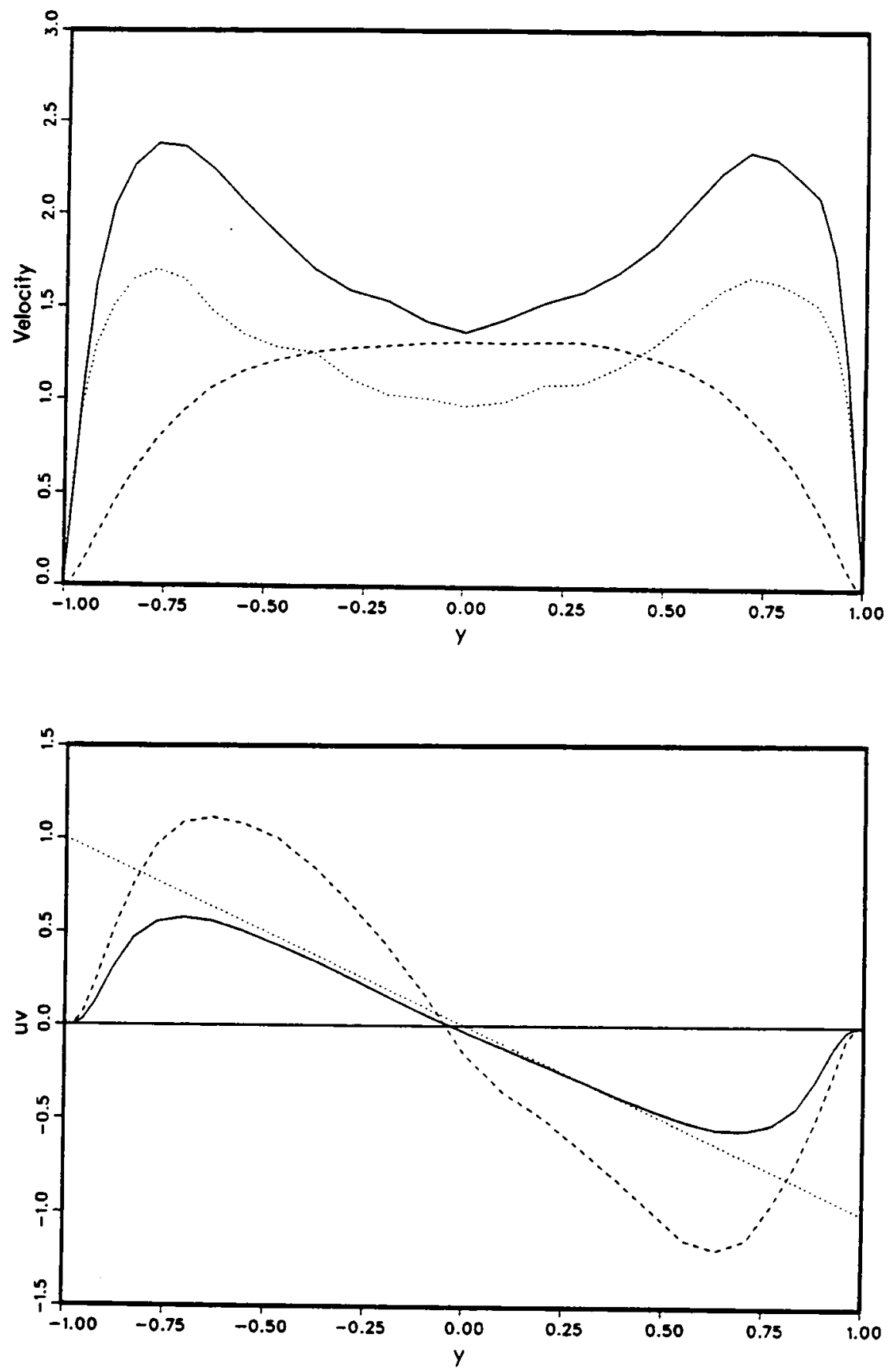

(c) r.m.s. velocities averaged over the wave area (full line $u_{\text {r.m.s., }}$ dashed line $v_{\text {r.m.s., }}$ and dotted line $w_{\text {r.m.s. }}$ ). (d) Reynolds shear stress in the turbulent (full line) and wave area (dashed line; dotted line is $u v=-y^{+}$). 

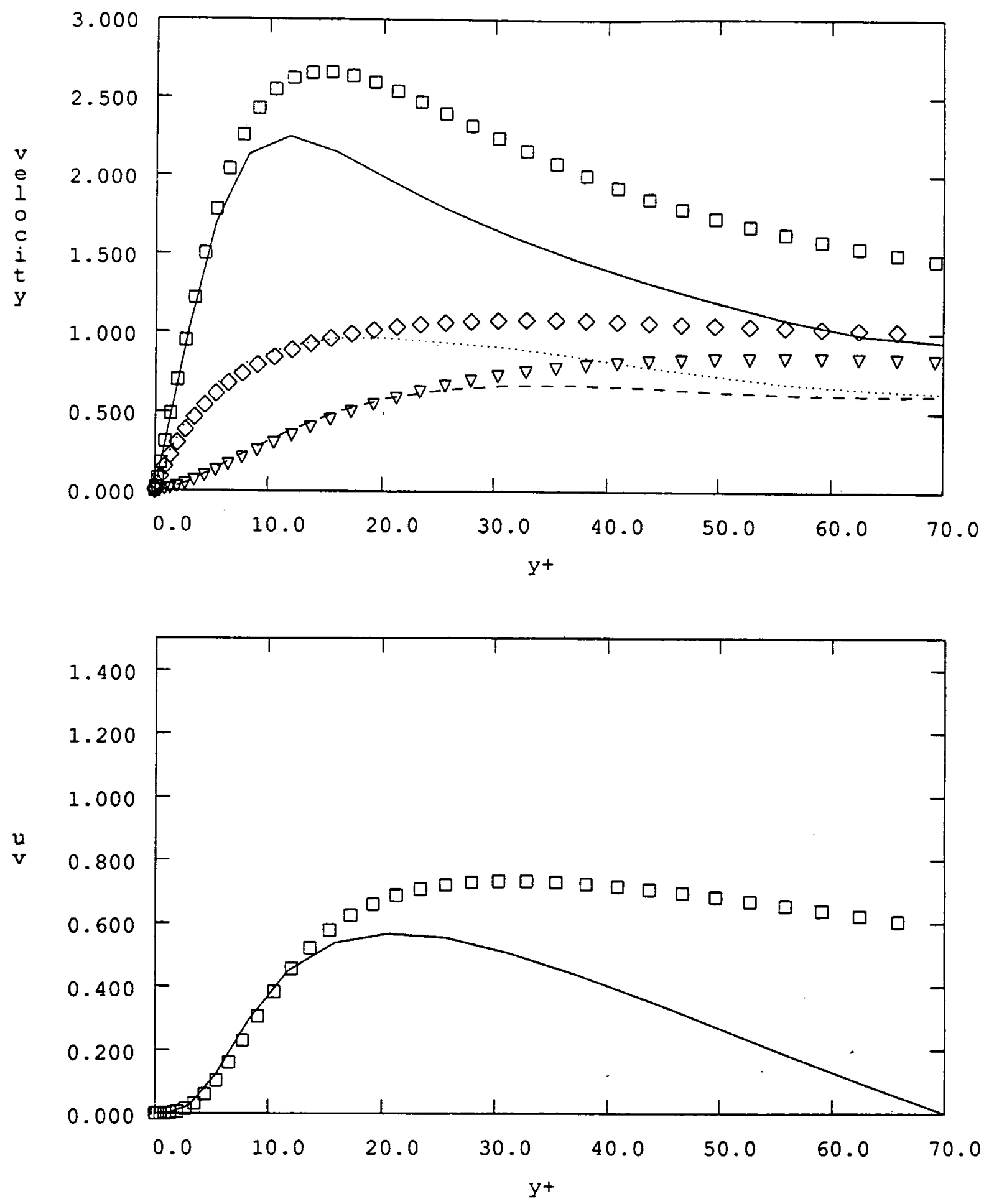

Figure 9. Comparison of normal dependence of turbulence quantities from the turbulent part of the spot (lines) with results from Kim, Moin, \& Moser (1987), (symbols). (a) R.m.s. velocities, ( $\Pi: u, \nabla: v, \diamond: w)$ (b) Reynolds stress, normal coordinate in wall units, 


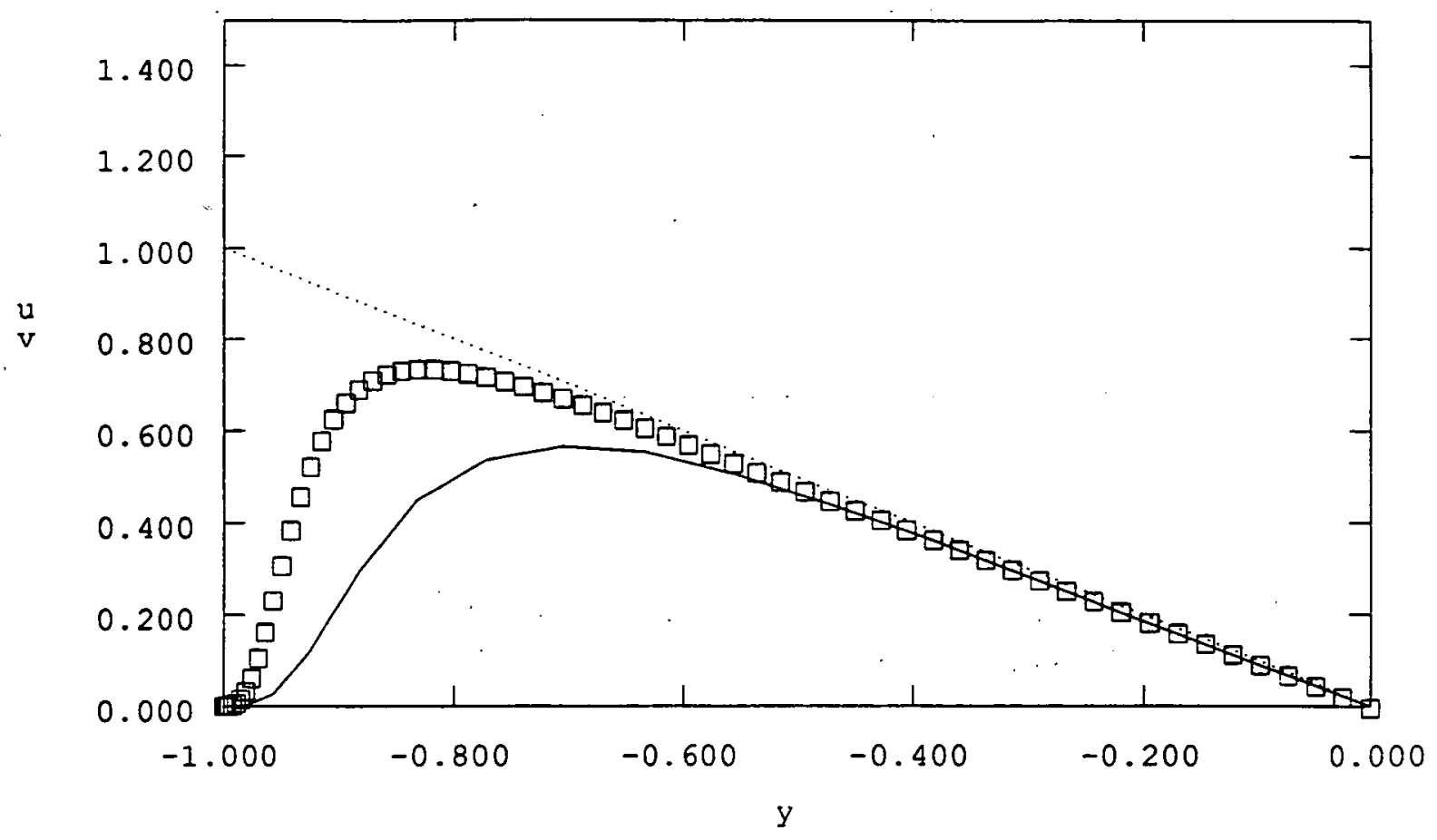

(c) Reynolds stress, normal coordinate in channel halfheights. Line types indicate the same velocities as in figure 8 . 


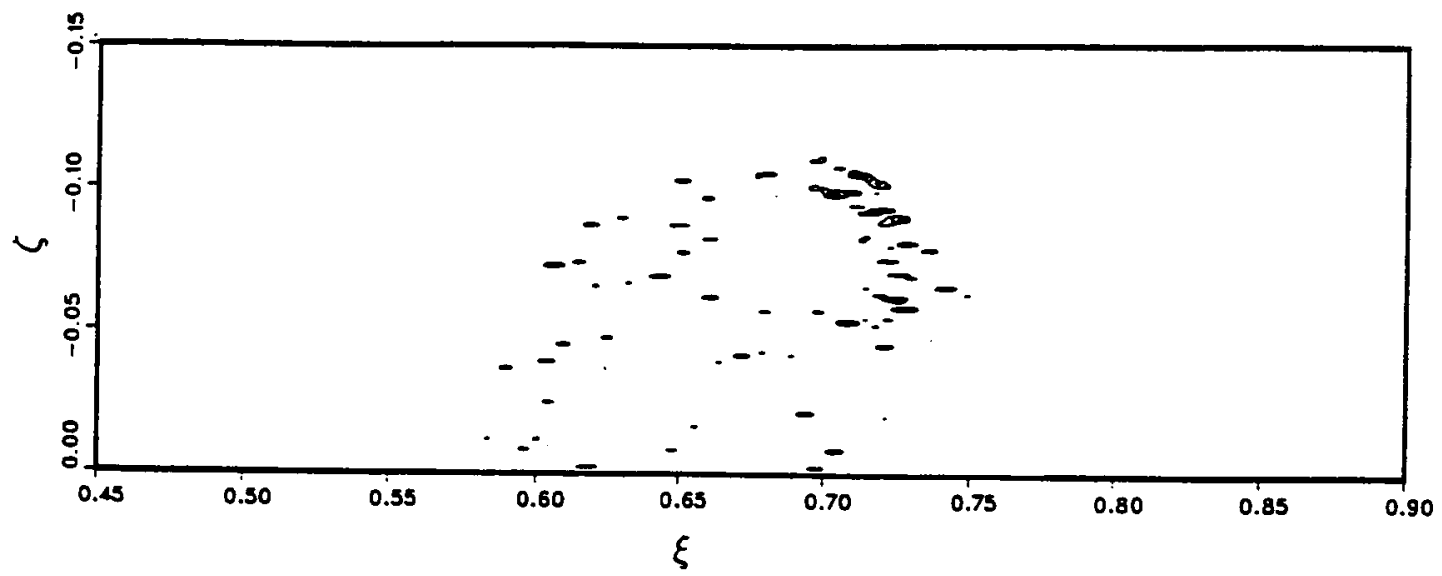

Figure 10. Islands of high variance obtained using the VISA technique, $y^{+}=12$. Contour spacing $k=0.5$, no zero contours plotted. 

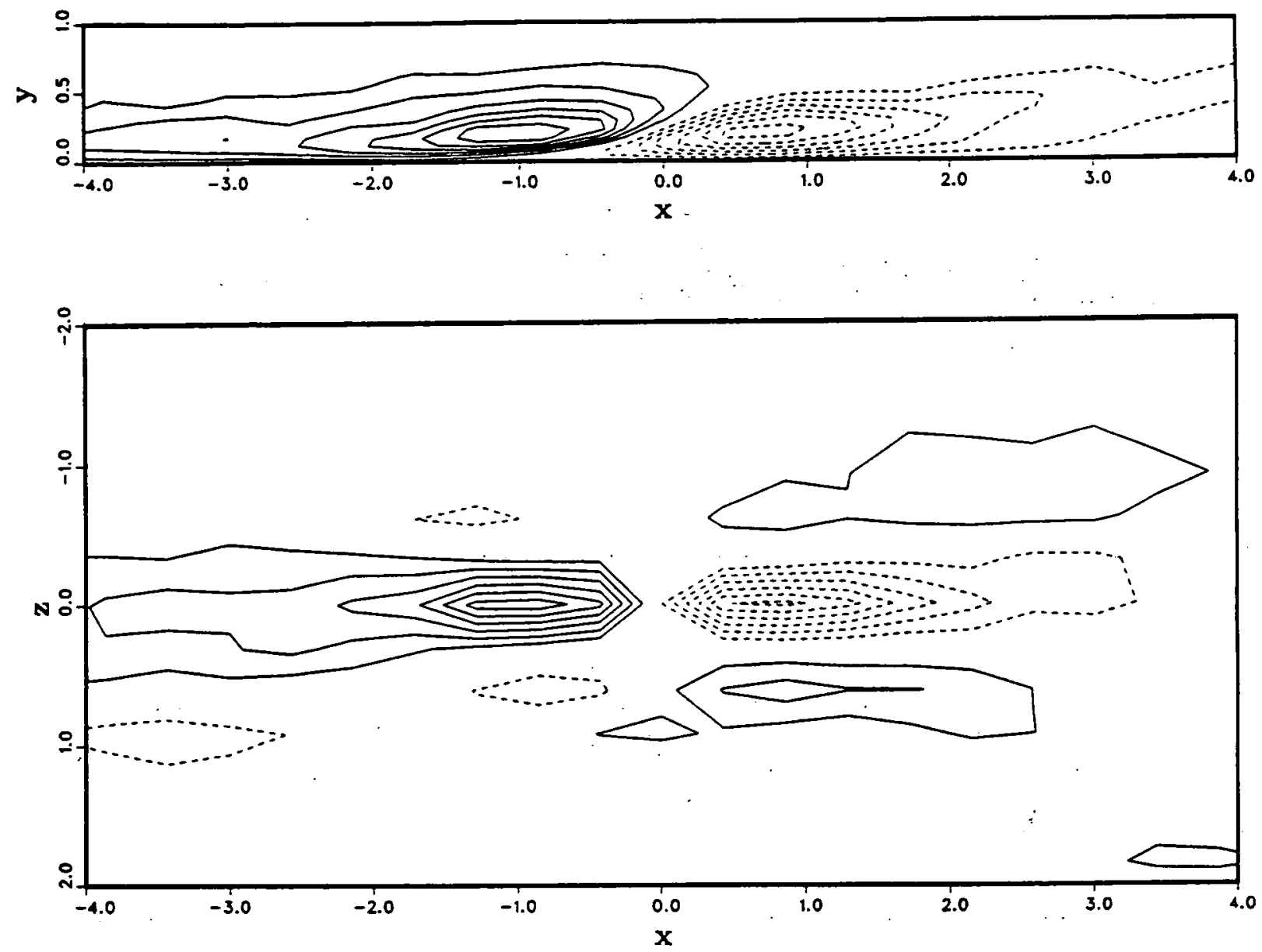

Figure 11. Contours of conditionally averaged velocities in the turbulent area. (a) Streamwise velocity in the $x-y$-plane along the centerline. (b) Streamwise velocity in the $x-z$-plane at $y=0.78$. The mean flow direction is from left to right, and the contour spacing is 0.025 or $0.5 u_{r}$. 

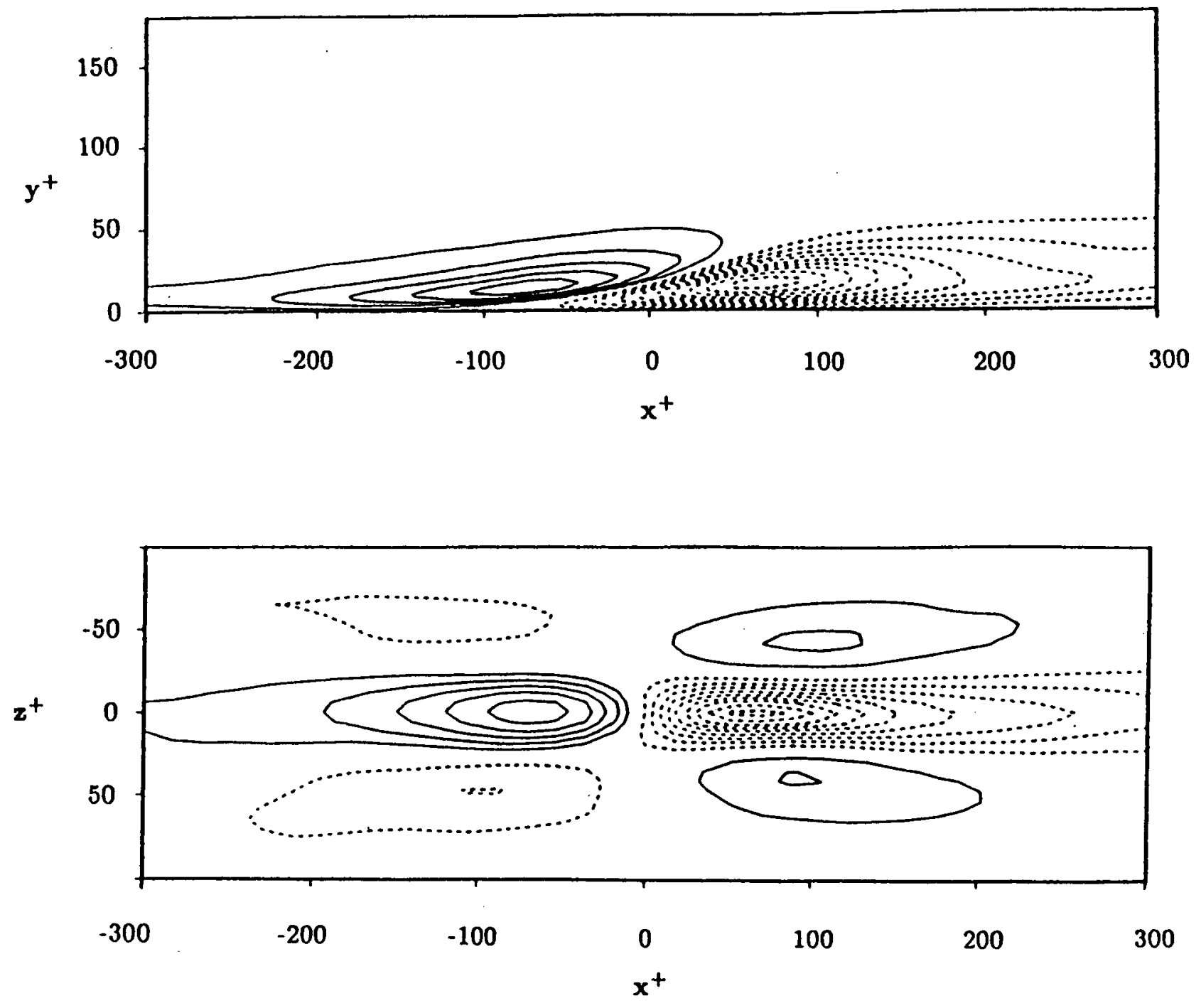

Figure 12. Contours of conditionally averaged velocities in fully developed turbulent channel flow. (a) Streamwise velocity in the $x-y$-plane along the centerline. (b) Streamwise velocity in the $x-z$-plane at $y^{+}=15$. The mean flow direction is from left to right, cont. spacing $0.5 u_{\tau}$. 

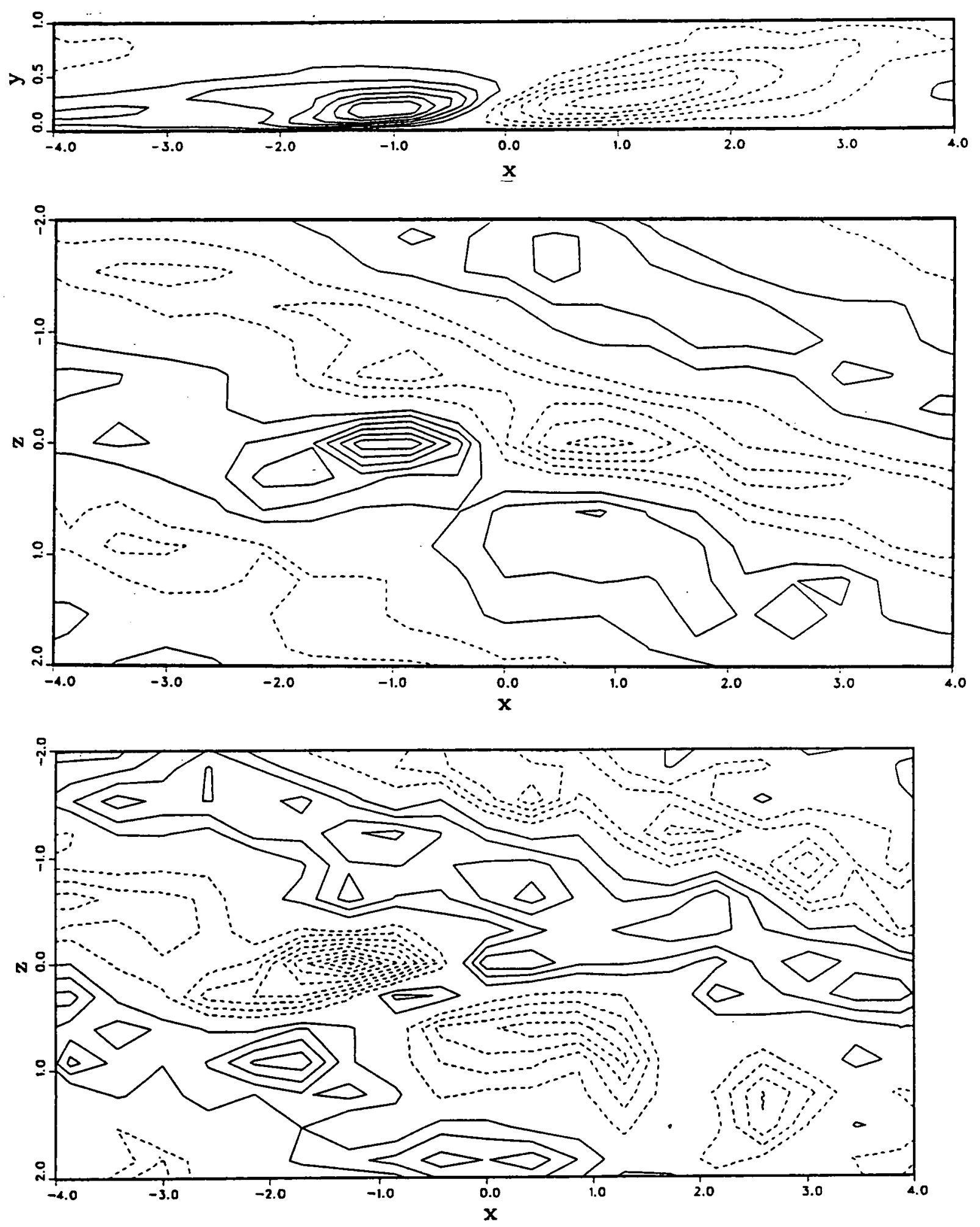

Figure 13. Contours of conditionally averaged velocities in the wave area. (a) Streamwise velocity in the $x-y$-plane along the centerline. (b) Streamwise velocity in the $x-z$-plane at $y=0.78$. (c) Normal velocity in the $x-z$-plane at $y=0.78$. The mean flow is from left to right. The the contour spacing for (a) and (b) is the same as in fig. 11 , and for (c) is 0.005 or $0.1 u_{\tau}$. 


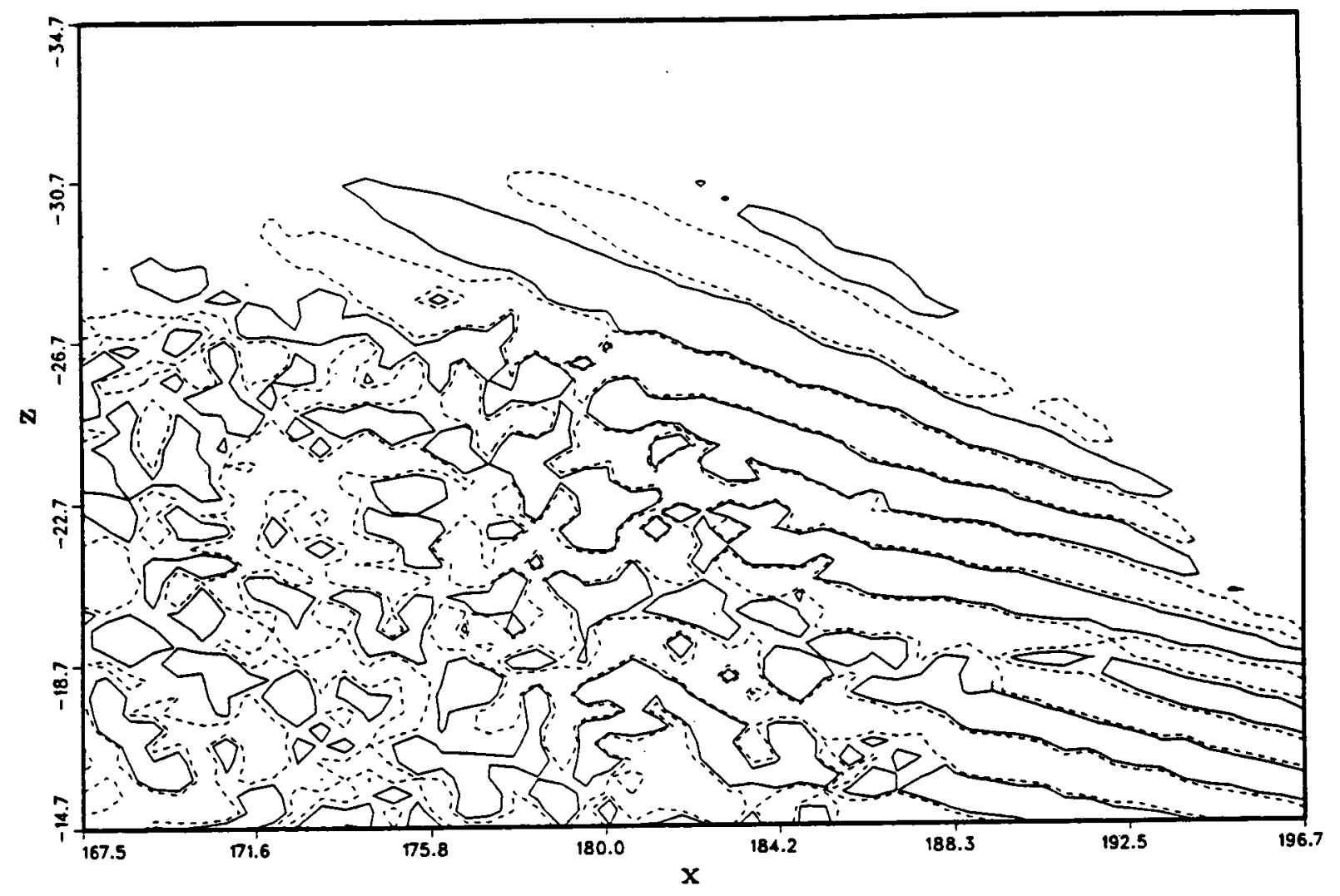

Figure 14. \pm 0.01 contours of instantaneous normal velocity at $y=0$ for $t=254.4$. 
Public reporting burden for this collection of information is estimated to average 1 hour per response, including the time tor reviewing instructions, searching existing data sources, gathering and maimtaining the data needed, and compteting and reviewing the collection of information. Send comments regarding this burden estimate or any other aspect of this collection of information, including suggestions for reducing this burden. to Washington Headquarters Services, Directorate for intormation Cperations and Report8, 1215 Jefterson Davis Highway. Suhte 1204. Arlington. VA 22202-4302, and to the Ottice of Managemem and Budget, Papenwork Reduction Project (0704-0188), Washington, OC 20503.

\begin{tabular}{l|l|l} 
1. AGENCY USE ONLY (Leave blank) & 2. REPORT DATE & 3. REPORT TYPE AND DATES COVERED
\end{tabular}

February 1992 Technical Memorandum

\section{TITLE AND SUBTITLE}

On Turbulent Spots in Plane Poiseuille Flow

6. AUTHOR(S)

Dan S. Henningson and John Kim

7. PERforming ORgaNizATION NAME(S) AND ADDRESS(ES)

8. PERForMING ORganization REPORT NUMBER

Ames Research Center

Moffett Field, CA 94035-1000

A-90192

9. SPONSORING/MONITORING AGENCY NAME(S) AND ADDRESS(ES)

10. SPONSOAING/MONITORING AGENCY REPORT NUMBER

National Aeronautics and Space Administration

Washington, DC 20546-0001

NASA TM-102836

11. SUPPLEMENTARY NOTES

Point of Contact: John Kim, Ames Research Center, MS 202A-1, Moffett Field, CA 94035-1000

(415) 604-5867 or FTS 464-5867.

To be published in the Journal of Fluid Mechanics.

Dan S. Henningson, Massachusetts Institute of Technology, Cambridge, Massachusetts

12a. DISTRIBUTION/AVAILABILITY STATEMENT

12b. DISTRIBUTION CODE

Unclassified - Unlimited

Subject Category 34

\section{ABSTRACT (Maximum 200 words)}

Turbulence characteristics inside a turbulent spot in plane Poiseuille flow are investigated by analyzing a database obtained from a direct numerical simulation. The spot is found to consist of two distinct regions-a turbulent area and a wave area. The flow inside the turbulent area has a strong resemblance to that found in the fully developed turbulent channel. Suitably defined mean and $\mathrm{ms}$ fluctuations as well as the intemal shear-layer structures are found to be similar to the turbulent counterpart. In the wave area the inflexional mean spanwise profiles cause a rapid growth of oblique waves, which break down to turbulence. The breakdown process of the oblique waves is reminiscent of the secondary instability observed during transition to turbulence in channel and boundary-layer flows. Other detailed characteristics associated with the Poiseuille spot are presented and are compared with experimental results.

\section{SUBJECT TERMS}

Turbulence, Turbulent spots, Transition

15. NUMBER OF PAGES

16. PRICE CODE

$\mathrm{A03}$

\begin{tabular}{|c|c|c|}
\hline $\begin{array}{l}\text { 17. SECUAITY CLASSIFICATION } \\
\text { OF REPORT }\end{array}$ & $\begin{array}{l}\text { 18. SECURITY CLASSIFICATION } \\
\text { OF THIS PAGE }\end{array}$ & $\begin{array}{l}\text { 19. SECURITY CLASSIFICATION } \\
\text { OF ABSTRACT }\end{array}$ \\
\hline Unclassified & Unclassified & \\
\hline
\end{tabular}

